\title{
THE STRUCTURE OF SEMIPRIMARY AND NOETHERIAN HEREDITARY RINGS
}

\author{
BY
}

JOHN FUELBERTH (1) AND JAMES KUZMANOVICH

\begin{abstract}
In the first portion of this paper a structure theorem for semiprimary hereditary rings is given in terms of $M \times M$ "triangular" row-finite matrices over a division ring $D$. This structure theorem differs from previous theorems of this type in that the representation is explicit in terms of matrices over a division ring. In the second portion of this paper we are able to apply the results of Gordon and Small to obtain a structure theorem for semihereditary and left hereditary rings which are left orders in a semiprimary ring. In the case of the left hereditary rings, the representation is explicit in terms of matrices over left hereditary Goldie prime rings and their respective classical left quotient rings. As an application we obtain, by a different method, a nonNoetherian generalization of a result of Chatters which states that a two-sided hereditary Noetherian ring is a ring direct sum of an Artinian ring and a semiprime ring.
\end{abstract}

1. Preliminaries. Throughout this paper all rings $R$ are assumed to be associative with multiplicative identity, and all $R$-modules will be unitary. A right or left $R$-module will be denoted by $M_{R}$ or ${ }_{R} M$ respectively. The socle of an $R$ module $M$ will be denoted by $\operatorname{soc}(M)$.

If $R$ is a ring, the Jacobson radical of $R$ will be denoted by $J(R)$, or just $J$ if there is no ambiguity. The prime radical of a ring $R$ is denoted by $N(R)$ or simply $N$. Semisimple shall mean semisimple Artinian. A ring $R$ is semiperfect [3] if $R / J$ is semisimple and idempotents can be lifted modulo $J$. A ring $R$ is semiprimary if $R$ is semiperfect and $J$ is nilpotent. In this case $N=J$.

The module $M$ is of finite (Goldie) dimension over $R$ if every direct sum of nonzero submodules of $M$ contains only a finite number of summands. We say that $R$ is left finite dimensional if ${ }_{R} R$ is of finite dimension.

If $R$ is left nonsingular, then $R$ possesses a maximal left quotient ring

Received by the editors April 26, 1974.

AMS (MOS) subject classifications (1970). Primary 16A48, 16A50; Secondary 16A42, 16A46, 16A08, 18G05.

Key words and phrases. Semiprimary ring, hereditary ring, semihereditary ring, Noetherian ring, nonsingular ring, regular ring, piecewise domain, Goldie prime ring, maximal quotient ring, classical quotient ring, row-finite matrices, flat module, projective module, uniform characteristic.

(1) This author was partially supported by a grant from the Research and Publications Committee at the University of Northern Colorado. 
(MLQ) $T$ which is a regular ring [21]. If $R$ is left finite dimensional, then the MLQ of $R$ is semisimple [28].

A nonzero element of a ring $R$ is regular if it has zero left and right annihilators. A ring $Q$ is said to be a classical left quotient ring of $R$ ( $R$ is called a left order in $Q$ ) if (i) $R \subset Q$, (ii) every element of $Q$ has the form $c^{-1} a$ where $a, c \in R$ with $c$ regular in $R$, and (iii) every regular element of $R$ has an inverse in $Q$.

For all homological notions, we refer the reader to [4].

Let $R$ be a semiperfect ring. Then $R=\bigoplus_{1 \leqslant i \leqslant n} \Sigma_{1 \leqslant j \leqslant m_{i}} R e_{i j}$ where the $e_{i j}$ are primitive idempotents and $R e_{i j} \simeq R e_{i^{\prime} j^{\prime}}$ if and only if $i=i^{\prime}$. Let $e=$ $e_{11}+\cdots+e_{n 1}$. Then the ring $e R e=R^{\prime}$ is a direct sum of pairwise nonisomorphic primitive left ideals and is semiperfect. $R^{\prime}$ is called the basic ring of $R$ and $R^{\prime}$ is unique up to isomorphism [15]. The ring $R$ may be recovered from the ring $R^{\prime}$. In fact, $R$ may be taken to be the ring of all $n \times n$ blocked matrices

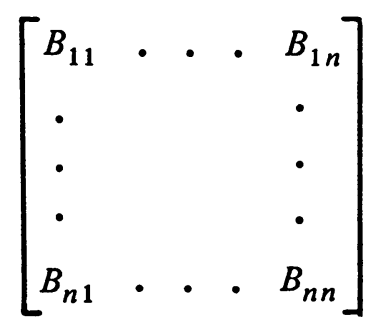

where $B_{i j}$ is the $e_{i} R^{\prime} e_{i}-e_{j} R^{\prime} e_{j}$ bimodule of $m_{i} \times m_{j}$ matrices with entries in $e_{i} R^{\prime} e_{j}$ [15]. Furthermore it is well known [15] that $R$ and $R^{\prime}$ are Morita equivalent. Hence we may reduce the study of the representation theory of semiperfect rings to that of semiperfect basic rings.

If $D$ is a division ring and if $I$ and $J$ are sets, then an $I \times J$ matrix over $D$ is a function from $I \times J$ to $D$. Multiplication of row-finite $I \times I$ matrices can be defined in a natural way, and there is the usual correspondence between linear transformations and matrices. The interested reader may consult Jacobson [20, Chapter IX].

A ring is said to have unmixed characteristic if as an abelian group it is $p$ primary or torsionfree. A ring $R$ is said to have uniform characteristic if every nonzero element of $R$ has the same additive order.

Proposition 1.1. Let $R$ be an indecomposable semiprimary ring. Then $R$ has unmixed characteristic.

Proof. If $(R,+)$ is a torsion group, then it has bounded order since $R$ has only finitely many nonisomorphic simple modules. Since the $p$-primary 
components of $(R,+)$ are ideals of $R$, the group primary decomposition of $R$ yields a ring decomposition of $R$. $R$ is indecomposable so that $R$ must have just one $p$-primary component and hence has unmixed characteristic. Hence we may assume that $(R,+)$ is not a torsion group.

Let $\left\{e_{1}, \ldots, e_{n}\right\}$ be a complete set of primitive idempotents for $R$. Consider a simple right module $S ; S$ is isomorphic to $e_{i} R / e_{i} N$ for some $i$. $S$ is a left vector space over the division ring $e_{i} R e_{i} / e_{i} N e_{i}$, and hence the characteristic of $S$ is the same as that of $e_{i} R e_{i} / e_{i} N e_{i}$. The ring $e_{i} R e_{i}$ is a local semiprimary ring whose only simple module is $e_{i} R e_{i} / e_{i} N e_{i}$ and hence $e_{i} R e_{i}$ has unmixed characteristic as does every module over $e_{i} R e_{i}$.

Let $e$ be the sum of those $e_{i}$ 's for which $e_{i} R / e_{i} N$ has characteristic zero and let $f=1-e$ be the sum of the remainder. The ring $e R e$ is a semiprimary ring over which every simple module has characteristic zero; hence every module over $e R e$ is torsionfree as an abelian group. The ring $f R f$, however, is a semiprimary ring over which every simple module has prime characteristic; hence every module over $f R f$ is torsion as an abelian group. This forces the bimodules $f R e$ and $e R f$ to be 0 . Thus $R$ is the ring direct sum of $e R e$ and $f R f$, and since $R$ is an indecomposable ring for which $(R,+)$ is not torsion, it must be that $R=e R e$ is torsionfree and $R$ has unmixed characteristic.

COROllaRy 1.2. A nonsingular indecomposable semiprimary ring has uniform characteristic.

Proof. If $R$ has characteristic zero, then the result follows from Proposition 1.1. If not, then by Proposition $1.1, R$ must have characteristic $p^{k}$. Let $Q$ be the maximal quotient ring of $R ; Q$ is semiprime. If $k \neq 1$, then the ideal ( $p 1) Q$ is nilpotent of index $k$ since $p 1$ is an element of the center of $Q$. This is a contradiction since $Q$ is semiprime; thus char $R=p$ and $R$ has uniform characteristic.

It is also true that an indecomposable PWD (see §2) has uniform characteristic, the proof being very similar to that of Proposition 1.1.

We will need the following result due to P. M. Cohn [10].

THEOREM 1.3. Two division rings $K, L$ can be imbedded in a common division ring if and only if they have the same characteristic.

2. Hereditary semiprimary rings. The purpose of this section is to prove Theorem 2.1 which describes the structure of a hereditary semiprimary ring $R$ in terms of $M \times M$ "triangular" row-finite matrices over a division ring $D$. There have been several results in the past (Chase [8], Harada [19], Gordon [15]) concerning triangular representation of a hereditary ring. Theorem 2.1 differs from 
these mainly in that it gives an explicit representation in terms of matrices over a division ring. The results of this section will be used in $\$ 3$, which is concerned with semihereditary and hereditary rings. Some of the results are broader than needed, but they will be of use in a subsequent paper on splitting rings.

THEOREM 2.1. Let $R$ be a basic indecomposable hereditary semiprimary ring. Then: (i) There is a division ring $D$. (ii) There are ordinal numbers $l_{1} \geqslant$ $l_{2} \geqslant \cdots \geqslant l_{n}\left(\right.$ let $\left.M=l_{1}+\cdots+l_{n}\right)$. (iii) For each $i=1, \ldots, n$ there is a division subring $D_{i}$ of the ring of $l_{i} \times l_{i}$ row-finite matrices over $D$. (iv) For each $i$ there is a sequence of integers $i<i(1)<i(2)<\cdots<i(k) \leqslant n$ and a sequence of ordinal numbers $v(1), v(2), \ldots, v(k)$ for which $\sum_{\alpha=1}^{k} l_{i(\alpha)} v(\alpha)=$ $l_{i}$; such that $R$ is isomorphic to the following blocked lower triangular subring of the ring $Q$ of $M \times M$ row-finite matrices over $D$ :

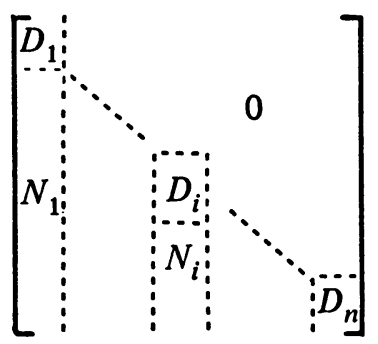

where each $N_{i}$ is defined recursively in terms of $N_{j}$ for $j>i$ by

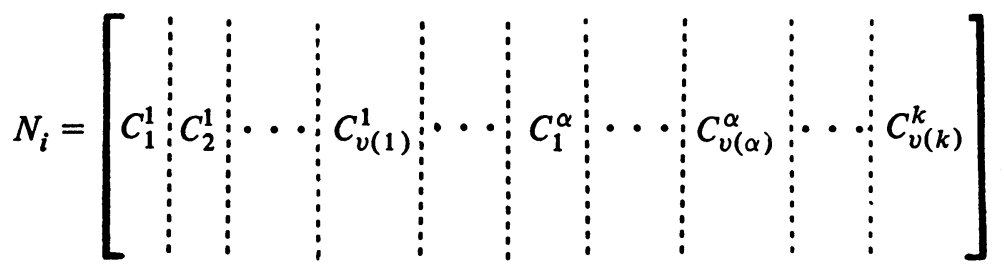

and where $C_{\beta}^{\alpha}$ is the collection of all matrices of the following form

$$
C_{\beta}^{\alpha}={ }^{t}\left[\begin{array}{l:l}
0 & D_{i(\alpha)} \\
N_{i(\alpha)}
\end{array}\right]
$$

where the zero denotes the appropriate sized zero matrix, and ${ }^{t}$ denotes transpose.

Conversely, any such matrix ring is a basic hereditary semiprimary ring and $Q$ contains a copy of the maximal left quotient ring of $R$.

The ring $R$ will be left Artinian exactly when the ordinal numbers $v(\alpha)$ are integers for each $i$.

As a simple example of the theorem let $R$ be the ring of matrices of the form 


$$
\left[\begin{array}{ccc}
a & 0 & 0 \\
0 & a & 0 \\
b & b+c & d
\end{array}\right]
$$

where $a, b, c$, and $d$ are real numbers. Then $R$ is an indecomposable hereditary semiprimary ring and $R$ is isomorphic to the ring $S$ of matrices of the form

$$
\left[\begin{array}{lll}
a & 0 & 0 \\
0 & a & 0 \\
b & c & d
\end{array}\right]
$$

The theorem states that there always is such an isomorphism.

The theorem is stated for an indecomposable ring since a semiprimary ring is a direct product of finitely many indecomposable semiprimary rings, and since a finite product of rings is hereditary if and only if each factor ring is hereditary. The theorem also assumes that $R$ is basic; in view of the results stated in $\S 1$ concerning basic rings and Morita equivalence this gives no loss of generality. While the assumption that $R$ is basic does not alter any ideas in the proof of Theorem 2.1 , it does help to simplify notation.

The remainder of this section is concerned with the proof of Theorem 2.1. The proof will consist of imbedding $R$ in a full $M \times M$ row-finite matrix $\operatorname{ring} Q$ over a division ring $D$ so that $Q$ is flat as a right $R$-module; using the flatness of $Q_{R}$ and the projectivity of left ideals of $R$ to draw conclusions concerning the matrices which represent $R$; and finally viewing $Q$ as the ring of linear transformations of $M$-dimensional row space over $D$ so that a change of basis can be used to obtain the desired representation for $R$.

PROPOSITION 2.2. Let $R$ be a semiprimary left nonsingular indecomposable ring. Then there is a cardinal number $M$ and a division ring $D$ such that $R$ can be imbedded in the ring $Q$ of $M \times M$ row-finite matrices over $D$ such that ${ }_{R} Q$ is nonsingular. If $R$ is left hereditary, then this can be done so that ${ }_{R} Q$ is $R$-injective and $Q_{R}$ is $R$-flat.

Proof. Since $R$ is left nonsingular and semiprimary by Johnson [22] the maximal left quotient ring $T$ of $R$ is a ring direct sum $T_{1} \times \cdots \times T_{k}$ where each $T_{i}$ can be identified with the $c_{i} \times c_{i}$ row-finite matrices over a division ring $K_{i}$. Let $M$ be the sum of the cardinal numbers $c_{1}, \ldots, c_{k}$. Since $R$ is indecomposable, $R$ has uniform characteristic by Corollary 1.2; hence, the division rings $K_{1}, \ldots, K_{k}$ have the same characteristic. Hence by Theorem 1.3 there is a divi- 
sion ring $D$ containing $K_{1}, \ldots, K_{k}$. Each $T_{i}$ is then a subring of the ring of $c_{i} \times c_{i}$ row-finite matrices over $D . T$ and $R$ are then imbedded in $Q$ by the map which imbeds the sum of the $c_{i} \times c_{i}$ matrix rings in $Q$ as a diagonal block matrix ring.

If $I$ is an essential left ideal of $R$ and if $q \in Q$ for which $I q=0$, then $T I q=$ 0 . The left $R$-module ${ }_{R} R$ is an essential submodule of ${ }_{R} T$ since $T=E\left({ }_{R} R\right)$; therefore, ${ }_{R} I$ is essential in ${ }_{R} T$, and finally $T I$ is thus an essential left ideal of $T$. As a result we have that ${ }_{R} Q$ is $R$-nonsingular if ${ }_{T} Q$ is $T$-nonsingular. Let $I$ be an essential left ideal of $T$ and let $q \in Q$ for which $I q=0$. Let $E_{h h}$ be the $M \times M$ matrix with a 1 in the $h, h$ position and zeroes elsewhere (for $h \in M$ ). The left ideal $T E_{h h}$ is a minimal left ideal of $T$; hence $T E_{h h}$ is contained in the essential left ideal $I$ and $E_{h h} \in I$. It follows that $E_{h h} q=0$ for all $h \in M$, and thus that $q=0$; that is, ${ }_{T} Q$ is nonsingular.

$R$ is hereditary semiprimary; therefore, by Cateforis $\left[5\right.$, p. 246] $T_{R}$ is flat. Since ${ }_{R} R$ is nonsingular, $T$ is a regular ring; hence it follows from $[4$, p. 123 , Problem 10] that $Q_{R}$ must be flat. Since $Q Q$ is $Q$-injective and since $Q_{R}$ is $R$ flat, we have that ${ }_{R} Q$ is $R$-injective, also by [4, p. 123, Problem 10].

A ring $R$ is called a PWD (see Gordon and Small [18]) if there is a complete set of orthogonal idempotents $\left\{e_{1}, \ldots, e_{n}\right\}$ with the property that $x y=0$ for $x \in e_{i} R e_{k}$ and $y \in e_{k} R e_{j}$ implies $x=0$ or $y=0$. All PWD's are nonsingular [17], and the class of PWD's properly includes the class of hereditary semiprimary rings.

Let $R$ be a basic semiprimary PWD with respect to the complete set of orthogonal idempotents $\left\{e_{1}, \ldots, e_{n}\right\}$. As in [18] we can order the idempotents $\left\{e_{1}, \ldots, e_{n}\right\}$ in a manner so that $e_{i} R e_{j}=\operatorname{Hom}_{R}\left(R e_{i}, R e_{j}\right)=0$ if $i<j$. Since $N$ is nilpotent, it also follows that $e_{i} N e_{i}=\operatorname{Hom}_{R}\left(R e_{i}, N e_{i}\right)=0$ and hence $e_{i} R e_{i}$ is a division ring $D_{i}$.

Let $K$ be a set and $D$ a division ring. A $K$-rspace ( $K$-cspace) over $D$ is the vector space over $D$ consisting of those $K$-rowtuples (columntuples) which are zero for all but finitely many $k$ in $K$.

By Proposition $2.2 R$ can be identified with a subring of the ring $Q$ of $M \times M$ row-finite matrices over a division ring $D$, and the $e_{i}$ 's will be a set of orthogonal idempotents of $Q$. The ring $Q$ is the ring of linear transformations of $M$-rspace over $D$; the set of idempotents $\left\{e_{1}, \ldots, e_{n}\right\}$ will then give a direct sum decomposition of $M$-rspace so that $e_{i}$ will be the natural projection on the $i$ th subspace. Choose a basis for the $i$ th subspace indexed by a set $A_{i}$; this can be done so that $A_{1} \cup \cdots \cup A_{n}=M$. Partially order $M$ so that if $x \in A_{i}$ and $y \in$ $A_{j}$ then $x<y$ if $i<j$. Represent each of the linear transformations with respect to the basis consisting of the union of the bases of the subspaces; $e_{i}$ will then be a matrix with an $A_{i} \times A_{i}$ identity block along the diagonal and zeroes elsewhere. In fact $R$ will then consist of a collection of matrices having the following form: 
$(*)$

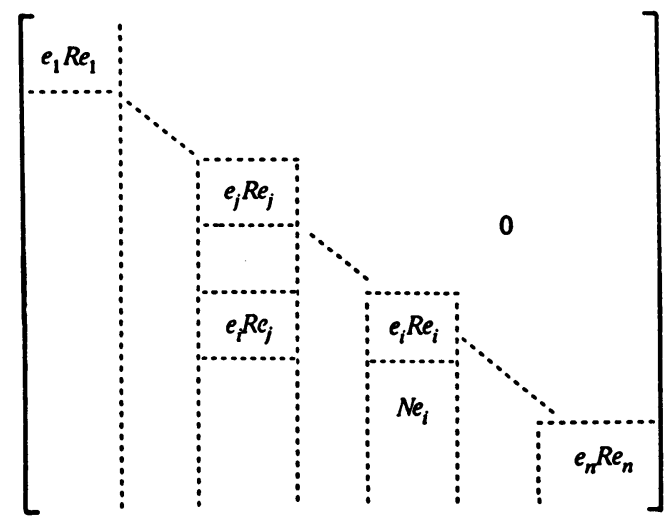

Since $R$ is basic, each $e_{i} R e_{i}$ can be identified (as in the diagram (*)) with a division subring of the ring of $A_{i} \times A_{i}$ row-finite matrices over $D$; as before denote it by $D_{i}$. Also, each $e_{i} R e_{j}$ can be naturally identified with a left $D_{i}$ (and right $D_{j}$ ) subspace of the space of $A_{i} \times A_{j}$ row-finite matrices over $D$. Throughout this section the elements of $e_{i} R e_{j}$ will be viewed both as $M \times M$ and $A_{i} \times A_{j}$ matrices.

We will need the following lemma which appears in Cartan-Eilenberg [4, p. 122, Problem 5].

LemMa 2.3. Let $R$ be a ring and let $A$ be a right $R$-module. If $I$ is a left ideal of $R$, then $A \otimes_{R} I \rightarrow A \otimes_{R} R$ is a monomorphism if and only if whenever $\Sigma a_{i} \mu_{i}=0$ for $\left\{a_{i}\right\} \subset A$ and $\left\{\mu_{i}\right\} \in I$, there exist $\left\{b_{i}\right\} \subset A$ and $\left\{\lambda_{i j}\right\} \subset R$ such that $\Sigma_{j} b_{j} \lambda_{i j}=a_{i}$ for all $i$ and $\Sigma_{i} \lambda_{i j} \mu_{i}=0$ for all $j$.

Let $E_{h k}$ be the $M \times M$ matrix with a 1 in the $h, k$ position and zeroes elsewhere. It follows that $Q=\Pi_{h \in M} E_{n h} Q$. Intuitively $E_{n h} Q$ is the $h$ th row of $Q$, while $Q E_{n h}$ is the $h$ th column.

The next three lemmas will be technical lemmas concerned with the flatness of $Q_{R}$.

Let $I$ and $J$ be sets. An $I \times J$ row-finite matrix will be called faithful if it is a monomorphism when considered as a linear transformation from $I$-rspace to $J$-rspace.

LEMma 2.4. Let $R$ be a PWD with structure as in (*). If $Q_{R}$ is $R$-flat, then: (1) if $x$ is a nonzero element of $e_{i} R e_{j}$, then $x$ is faithful; (2) if $e_{i} R e_{j} \neq$ 0 and $j<i$, then $\left|A_{i}\right| \leqslant\left|A_{j}\right|$; (3) the $e_{i}$ 's can be reordered in a manner consistent with the structure in $(*)$ so that $\left|A_{j}\right| \geqslant\left|A_{i}\right|$ whenever $j<i$.

Proof. (1) Suppose that $x$ is a nonzero element of $e_{i} R e_{j}$ and suppose that $q x=0$ for $0 \neq q \in A_{i}$-space. Identify $q$ with that element of $E_{a a} Q$ for which $q e_{i}=q$ where $a \in A_{i}$. Since $E_{a a} Q$ is flat, it follows from Lemma 2.3 that there 
are elements $\left\{b_{\alpha}\right\} \subset E_{a a} Q$ and $\left\{\lambda_{\alpha}\right\} \subset R$ for which $\Sigma b_{\alpha} \lambda_{\alpha}=q$ and $\lambda_{\alpha} x=0$ for all $\alpha$. Since $q=q e_{i} \neq 0$, for at least one $\alpha$, say $\alpha_{0}, \lambda_{\alpha_{0}} e_{i} \neq 0$. Since $R e_{i}$ is indecomposable and since $R$ is a PWD, we have that every element of $\operatorname{Hom}_{R}\left(R e_{i}, R e_{j}\right)$ is a monomorphism. Right multiplication by $x$ is such a homomorphism; therefore $\left(\lambda_{\alpha_{0}} e_{i}\right) x \neq 0$. But this is a contradiction since $\left(\lambda_{\alpha_{0}} e_{i}\right) x=$ $\lambda_{\alpha_{0}}\left(e_{i} x\right)=\lambda_{\alpha_{0}} x=0$. Hence there can be no such $q$ and $x$ is faithful.

(2) Let $x$ be a nonzero element of $e_{i} R e_{j}$. Consider $x$ as a linear transformation from $A_{i}$-rspace to $A_{j}$-rspace. By (1) dim range $x=\left|A_{i}\right|$ which then must be less than $\left|A_{j}\right|$.

(3) Suppose that the $e_{i}$ 's are not ordered in the desired manner; then there is an $i$ for which $\left|A_{i}\right|<\left|A_{j}\right|$ for some $j>i$. Let $i$ be the first such $i$ and let $j$ be the first such $j$. Let $i \leqslant k<j$, then $\left|A_{j}\right|>\left|A_{i}\right| \geqslant\left|A_{k}\right|$; hence by (1) $\operatorname{Hom}_{R}\left(R e_{j}, R e_{k}\right)=0$. As a result the ordering $\left\{e_{1}, \ldots, e_{i-1}, e_{j}, e_{i}, \ldots, e_{j-1}\right.$, $\left.e_{j+1}, \ldots, e_{n}\right\}$ will retain the property that $e_{k} R e_{m}=0$ whenever $m>k$. This reordering of the $e_{i}$ 's will retain the structure (*). Continuing this process will give the desired ordering.

For the rest of this section assume that the $e_{i}$ 's are ordered as in Lemma 2.4 (3).

Lemma 2.5. Let $R$ be semiprimary. A right $R$-module $A_{R}$ is flat if and only if $0 \rightarrow A \otimes_{R} N e_{i} \rightarrow A \otimes_{R} R e_{i}$ is exact for all $i$.

Proof. The ring $R$ is semiprimary so that by Proposition 7 of Auslander [2], $A_{R}$ will be flat if and only if $\operatorname{Tor}_{1}^{R}\left(A_{R}, S_{R}\right)=0$ for all simple right $R$ modules $S_{R}$. The set $\left\{R e_{1} / N e_{1}, \ldots, R e_{n} / N e_{n}\right\}$ is a complete set of representatives for the isomorphism classes of simple right modules over $R$; therefore, by a long exact sequence of Tor we have that $A_{R}$ is flat if and only if $0 \rightarrow A \otimes$ $N e_{i} \rightarrow A \otimes R e_{i}$ is exact for all $i$.

Let $\left\{V_{\alpha}=\alpha \in A\right\}$ be a family of vector spaces over a division ring $D$ and let $T_{\alpha} \in \operatorname{Hom}\left(V_{\alpha}, V\right)$ for some vector space $V$ over $D$. The family $\left\{T_{\alpha}\right\}$ will be called codirect if their images sum directly as subspaces of $V$. The family $\left\{T_{\alpha}\right\}$ will be called full if the sum of their images is all of $V$. A family of matrices will be called codirect (full) if the family of linear transformations they represent is codirect (full). A family of matrices will be called faithful if each matrix in the family is faithful.

The following lemma will give a criterion for the codirectness of a family of matrices.

LEMMA 2.6. Let $A$ be a well-ordered set and let $\left\{x_{\alpha}: \alpha \in A\right\}$ be a faithful family of $A_{\alpha} \times M$ row-finite matrices where the $A_{\alpha}$ 's and $M$ are sets. The family $\left\{x_{\alpha}\right\}$ will be codirect if and only if the matrix $C$ 


$$
C=\left[\begin{array}{c}
x_{1} \\
\cdots . . . \\
x_{2} \\
\hdashline \\
\bullet
\end{array}\right]
$$

is a faithful linear transformation from $\bigoplus_{\alpha \in A}\left(A_{\alpha}\right.$-rspace) to M-rspace.

PROOF. The $\left\{x_{\alpha}\right\}$ are codirect if and only if $\Sigma q_{\alpha} x_{\alpha}=0$ for $q_{\alpha} \in A_{\alpha^{-}}$ rspace implies each $q_{\alpha} x_{\alpha}=0$. Since each $x_{\alpha}$ is faithful, this happens exactly when each $q_{\alpha}$ is zero.

The following proposition will constitute the main part of the proof of Theorem 2.1.

Proposition 2.7. Let $R$ be a semiprimary ring with matrix structure as in (*). The ring $R$ will be hereditary with $Q_{R}$ flat if and only if for each $i, N e_{i}$ has a faithful codirect family of generators.

Proof. Since $R$ is semiprimary and $\operatorname{Hom}\left(R e_{j}, N e_{i}\right)=0$ for $j \leqslant i$, it follows that for each $i$ there is a sequence of positive integers $i<i(1)<i(2)<\cdots<$ $i(k) \leqslant n$ and a set $A_{\alpha}$ for $\alpha=1, \ldots, k$ such that $N e_{i} / N^{2} e_{i} \simeq \bigoplus\left\{\left(R e_{i(\alpha)} / N e_{i(\alpha)}\right)^{A} \alpha\right.$ : $\alpha=1, \ldots, k\}$ where $\left(R e_{i(\alpha)} / N e_{i(\alpha)}\right)^{A} \alpha$ denotes the direct sum of copies of $R e_{i(\alpha)} / N e_{i(\alpha)}$ indexed by the set $A_{\alpha}$. It follows then that $N e_{i}$ has a set of generators $\left\{u_{\beta}^{\alpha}\right\}$ where $u_{\beta}^{\alpha} \in e_{i(\alpha)} R e_{i}$ and $R u_{\beta}^{\alpha} / N u_{\beta}^{\alpha} \simeq R e_{i(\alpha)} / N e_{i(\alpha)}$ for $\alpha=1, \ldots, k$ and $\beta \in A_{\alpha}$.

$\Leftrightarrow$ Let $R$ be hereditary and let $Q_{R}$ be flat. Consider $N e_{i}$ and $\left\{u_{\beta}^{\alpha}\right\}$ chosen as above. The projective cover of $N e_{i} / N^{2} e_{i}$ is isomorphic to $P=\bigoplus\left(R e_{i(\alpha)}\right)^{A}$. Since $R$ is hereditary, $N e_{i}$ is also a projective cover of $N e_{i} / N^{2} e_{i}$ and it follows easily from a natural isomorphism of $P$ onto $\Sigma R u_{\beta}^{\alpha}$ that the $R u_{\beta}^{\alpha}$ 's sum directly.

To show $\left\{u_{\beta}^{\alpha}\right\}$ is codirect, suppose that there are $q_{\beta}^{\alpha}$ s in $A_{\alpha}$-rspace for which $\Sigma_{\alpha, \beta} q_{\beta}^{\alpha} u_{\beta}^{\alpha}=0$. Identify each $q_{\beta}^{\alpha}$ in the natural manner with that element of $E_{m m} Q$ for which $q_{\beta}^{\alpha} e_{i(\alpha)}=q_{\beta}^{\alpha}$ for some fixed $m$ in $M$. The module $Q_{R}$ is flat, hence so is $E_{m m} Q$; therefore by Lemma 2.3 there exist $b_{\gamma}$ 's in $E_{m m} Q$ and $\left\{\lambda_{\beta \gamma}^{\alpha}\right\} \subset R$ such that $\Sigma_{\gamma} b_{\gamma} \lambda_{\beta \gamma}^{\alpha}=q_{\beta}^{\alpha}$ for each $\beta$ and $\alpha$, and $\Sigma_{\alpha, \beta} \lambda_{\beta \gamma}^{\alpha} u_{\beta}^{\alpha}=0$ for all $\gamma$. Since the $R u_{\beta}^{\alpha}$ 's sum directly, $\lambda_{\beta \gamma}^{\alpha} u_{\beta}^{\alpha}=0$ for all $\alpha, \beta, \gamma$. Since $e_{i(\alpha)} u_{\beta}^{\alpha}$ $=u_{\beta}^{\alpha}$, we have that $\left(\lambda_{\beta \gamma}^{\alpha} e_{i(\alpha)}\right) u_{\beta}^{\alpha}=\lambda_{\beta \gamma}^{\alpha}\left(e_{i(\alpha)} u_{\beta}^{\alpha}\right)=\lambda_{\beta \gamma}^{\alpha} u_{\beta}^{\alpha}=0$. Each $u_{\beta}^{\alpha}$ is faithful by Lemma 2.4; this implies $\lambda_{\beta \gamma}^{\alpha} e_{i(\alpha)}=0$ for all $\alpha, \beta, \gamma$. Therefore

$$
q_{\beta}^{\alpha}=q_{\beta}^{\alpha} e_{i(\alpha)}=\left(\sum_{\gamma} b_{\gamma} \lambda_{\beta \gamma}^{\alpha}\right) e_{i(\alpha)}=\sum_{\gamma} b_{\gamma} \lambda_{\beta \gamma}^{\alpha} e_{i(\alpha)}=0 .
$$


Thus all the $q_{\beta}^{\alpha}$ 's are zero, and the family $\left\{u_{\beta}^{\alpha}\right\}$ must be codirect.

$\Leftrightarrow$ By Proposition 7 of Auslander [2] and by Lemma 2.5 it will be enough to show that for each $i, N e_{i}$ is projective and $0 \rightarrow Q \otimes N e_{i} \rightarrow Q \otimes R e_{i}$ is exact.

Consider $N e_{i}$ and let $\left\{u_{\beta}^{\alpha}\right\}$ as above, be a faithful codirect family. The family is faithful so each $u_{\beta}^{\alpha}$ is faithful and $R u_{\beta}^{\alpha}=\left(R e_{i(\alpha)}\right) u_{\beta}^{\alpha} \simeq R e_{i(\alpha)}$ is projective. Then $N e_{i}$ will be projective provided it can be shown that the $R u_{\beta}^{\alpha}$ 's sum directly. Let $0=\Sigma_{\alpha, \beta} r_{\beta}^{\alpha} u_{\beta}^{\alpha}$ for $\left\{r_{\beta}^{\alpha}\right\} \subset R$. This will be the case if and only if $E_{m m}\left(\Sigma r_{\beta}^{\alpha} u_{\beta}^{\alpha}\right)=0$ for all $m \in M$; but

$$
E_{m m}\left(\sum r_{\beta}^{\alpha} u_{\beta}^{\alpha}\right)=\sum_{\alpha, \beta} E_{m m} r_{\beta}^{\alpha} u_{\beta}^{\alpha}=\sum E_{m m_{\beta}} r_{\beta}^{\alpha} e_{i(\alpha)} u_{\beta}^{\alpha}=0
$$

since $e_{i(\alpha)} u_{\beta}^{\alpha}=u_{\beta}^{\alpha}$. For each $\alpha$ and $\beta, E_{m m} r_{\beta}^{\alpha} e_{i(\alpha)}$ can be identified with an element of $A_{\alpha}$-rspace; hence $E_{m m} r_{\beta}^{\alpha} e_{i(\alpha)}=0$ since $\left\{u_{\beta}^{\alpha}\right\}$ is codirect. Thus we have $0=r_{\beta}^{\alpha} e_{i(\alpha)}$ and $r_{\beta}^{\alpha} e_{i(\alpha)} u_{\beta}^{\alpha}=r_{\beta}^{\alpha} u_{\beta}^{\alpha}=0$ for all $a$ and $\beta$; hence the $R u_{\beta}^{\alpha}$ 's sum directly and $N e_{i}$ is projective. This shows that $R$ is left (and right) hereditary.

Since $R$ is hereditary and since $Q_{R}=\Pi_{m \in M} E_{m m} Q_{R}, Q_{R}$ will be flat if and only if $E_{m m} Q_{R}$ is flat for all $m$ by Theorem 2.1 of Chase [7]. The $R u_{\beta}^{\alpha}$ 's sum directly; therefore, every element of $E_{m m} Q \otimes N e_{i}$ can be written uniquely in the form $\Sigma_{\alpha, \beta} E_{m m} q_{\beta}^{\alpha} \otimes u_{\beta}^{\alpha}$. Suppose that $\Sigma_{\alpha, \beta} q_{\beta}^{\alpha} u_{\beta}^{\alpha}=0$ where $q_{\beta}^{\alpha} \in$ $E_{m m} Q$; then $0=\Sigma_{\alpha, \beta} q_{\beta}^{\alpha} u_{\beta}^{\alpha}=\Sigma q_{\beta}^{\alpha} e_{i(\alpha)} u_{\beta}^{\alpha}$ since $e_{i(\alpha)} u_{\beta}^{\alpha}=u_{\beta}^{\alpha}$. Each $q_{\beta}^{\alpha} e_{i(\alpha)}$ however can be naturally identified with an element of an $A_{\alpha}$-rspace; in which case $q_{\beta}^{\alpha} u_{\beta}^{\alpha}=q_{\beta}^{\alpha} e_{i(\alpha)} u_{\beta}^{\alpha}=0$ for each $\alpha$ and $\beta$ since $\left\{u_{\beta}^{\alpha}\right\}$ is codirect. Since each $u_{\beta}^{\alpha}$ is faithful, we further have that $q_{\beta}^{\alpha} e_{i(\alpha)}=0$ for each $\alpha$ and $\beta$. Let $b_{\beta}^{\alpha}=q_{\beta}^{\alpha}$ and let $\lambda_{\beta}^{\alpha}=1-e_{i(\alpha)}$. Then we have

$$
b_{\beta}^{\alpha} \lambda_{\beta}^{\alpha}=q_{\beta}^{\alpha}\left(1-e_{i(\alpha)}\right)=q_{\beta}^{\alpha}-q_{\beta}^{\alpha} e_{i(\alpha)}=q_{\beta}^{\alpha}-0=q_{\beta}^{\alpha}
$$

also,

$$
\lambda_{\beta}^{\alpha} u_{\beta}^{\alpha}=\left(1-e_{i(\alpha)}\right) u_{\beta}^{\alpha}=u_{\beta}^{\alpha}-e_{i(\alpha)} u_{\beta}^{\alpha}=u_{\beta}^{\alpha}-u_{\beta}^{\alpha}=0
$$

Therefore

$$
\sum_{\alpha, \beta} q_{\beta}^{\alpha} \otimes u_{\beta}^{\alpha}=\sum_{\alpha, \beta} b_{\beta}^{\alpha} \lambda_{\beta}^{\alpha} \otimes u_{\beta}^{\alpha}=\sum b_{\beta}^{\alpha} \otimes \lambda_{\beta}^{\alpha} u_{\beta}^{\alpha}=\sum b_{\beta}^{\alpha} \otimes 0=0
$$

and hence $0 \rightarrow E_{m m} Q \otimes N e_{i} \rightarrow E_{m m} Q \otimes R e_{i}$ is exact. Thus $E_{m m} Q_{R}$ is flat and hence $Q_{R}$ is flat.

We can now give the proof to Theorem 2.1.

Proof. Let $R$ be an indecomposable hereditary basic semiprimary ring. By Proposition 2.2 we can imbed $R$ with triangular structure (*) in the ring $Q$ of $M \times M$ row-finite matrices over a division ring $D$. Choose for each $i$ a codirect family of generators for $N e_{i}$ as in Proposition 2.7. Let $V$ be $M$-space over $D$; we 
will view $Q$ as the ring of linear transformations of $V$, and then choose a basis for $V$ which will give each $u_{\beta}^{\alpha}$ a representation which will then give $R$ the representation of Theorem 2.1.

The orthogonal set of idempotents $\left\{e_{1}, \ldots, e_{n}\right\}$ gives a direct sum decomposition of $V ; V=V e_{1} \oplus \cdots \oplus V e_{n}$. We will inductively choose a basis for each $V e_{i}$.

Choose any basis for $V e_{n}$. Well-order this basis and let its order type be that of an ordinal $l_{n}$.

Inductively assume that a basis of order type $l_{j}$ has been chosen for all $j>i$. Using the notation of Proposition 2.7, well-order each $A_{\alpha}$ and let the order type of $A_{\alpha}$ be $v(\alpha)$. The set $\left\{u_{\beta}^{\alpha}\right\}$ will then be well ordered under the lexicographic order. By Proposition 2.6 the matrix

$$
C={ }^{t}\left[u_{1}^{1}: \cdots: u_{v(k)}^{k}\right]
$$

will represent a faithful linear transformation from $U=\bigoplus_{\alpha, \beta} V e_{i(\alpha)}^{(\beta)}$ into $V e_{i}$ where $i<i(1)<\cdots<i(k) \leqslant n$ and where $V e_{i(\alpha)}^{(\beta)}$ denotes a copy of $V e_{i(\alpha)}$. Let $B_{\beta}^{\alpha}$ be the copy in $V e_{i(\alpha)}^{(\beta)}$ of the basis chosen for $V e_{i(\alpha)} ; B=\bigcup_{\alpha, \beta} B_{\beta}^{\alpha}$ will then be a basis for $U$, and if $B$ is ordered lexicographically it will have order type $\Sigma l_{i(\alpha)} v(\alpha)$. Since $C$ is faithful, $(B) C$ will be a linearly independent subset of $V e_{i}$; extend it to an ordered basis in a manner which extends the ordering of $(B) C$ induced by that of $B$. Denote the resulting order type by $l_{i}$. Continuing this process will give an ordered ba. sis for $V$; as a result we can consider $M$ to be the ordinal $\Sigma_{i=1}^{n} l_{i}$.

We will now show that $R$ will have the desired representation with respect to this basis. The structure of (1) follows from the fact that $R$ has a structure as in (*). Consider $N e_{i}$ and inductively assume that $N e_{j}$ has structure in (2) and (3) for all $j>i$. Let $L=\Sigma l_{i(\alpha)} v(\alpha)$. With respect to this basis $C$ will have the form $[I: 0]$ where $I$ is an $L \times L$ identity matrix. In this case $u_{\beta}^{\alpha}$ (considered as an $l_{i(\alpha)} \times l_{i}$ matrix) will have the form $[W \vdots I \vdots Z]$ where $W$ is an $l_{i(\alpha)} \times$ $\left(\Sigma_{\gamma<\alpha} l_{i(\gamma)} v(\gamma)+l_{i(\alpha)}(\beta-1)\right)$ zero matrix, $I$ is an $l_{i(\alpha)} \times l_{i(\alpha)}$ identity matrix, and $Z$ is the zero matrix consisting of the remaining columns. It is now easy to see that $R$ has the desired representation. Let $C_{\beta}^{\alpha}=R e_{i(\alpha)} u_{\beta}^{\alpha} ; u_{\beta}^{\alpha}$ is an $l_{i(\alpha)} \times l_{i(\alpha)}$ identity matrix so that $C_{\beta}^{\alpha}$ consists precisely of all matrices over $R e_{i(\alpha)}$. Hence $C_{\beta}^{\alpha}$ has the structure indicated in (3). By the above representation of $u_{\beta}^{\alpha}$ we see that $u_{\beta}^{\alpha}$ 's form a sequence of nonoverlapping subidentity matrices from left to right; therefore, the $C_{\beta}^{\alpha}$ 's are positioned as in (2). We have

$$
N e_{i}=\bigoplus_{\alpha, \beta} R u_{\beta}^{\alpha} \doteq \bigoplus_{\alpha, \beta} R e_{i(\alpha)} u_{\beta}^{\alpha}=\bigoplus_{\alpha, \beta} C_{\beta}^{\alpha}
$$

so that the $C_{\beta}^{\alpha}$ 's will represent everything in $N e_{i}$. Suppose now that $\sum_{\alpha=1}^{k} l_{i(\alpha)} v(\alpha) \neq l_{i}$; then the right-hand column of $N e_{i}$ will be identically zero. 
Suppose that this is the $m$ th column. We will then have

$$
\left(\operatorname{Soc}_{R} R\right) E_{m m} Q=\left(\left(\operatorname{Soc}_{R} R\right) E_{m m}\right) Q \subset\left(N e_{i}\right) E_{m m} Q=0
$$

since $e_{j} E_{m m}=0$ for $j \neq i$; but this contradicts that ${ }_{R} Q$ is nonsingular as in Proposition 2.2. We have thus shown that each $N e_{i}$ has the desired structure.

Conversely, assume $R$ is a ring with structure as in (1), (2) and (3). For each $i$ pick a family $\left\{u_{\beta}^{\alpha}\right\}$ where $u_{\beta}^{\alpha}$ is the $l_{i(\alpha)} \times l_{i(\alpha)}$ subidentity matrix of $C_{\beta}^{\alpha}$ for which $e_{i(\alpha)} u_{\beta}^{\alpha}=u_{\beta}^{\alpha}\left(e_{i(\alpha)}\right.$ is the identity element of $\left.D_{i(\alpha)}\right)$. By choice $\left\{u_{\beta}^{\alpha}\right\}$ will be a faithful codirect family of generators for $\mathrm{Ne}_{i}$. Hence, by Proposition $2.7, R$ will be hereditary and $Q_{R}$ will be flat. It is easy to see that $N$ is nilpotent of index at most $n$, so that $R$ is semiprimary.

We show that the maximal left quotient ring $T$ of $R$ is a subring of $Q$. If $q \in$ $Q$ for which $\operatorname{Soc}\left(R e_{n}\right) q=\left(R e_{n}\right) q=0$, then clearly $e_{n} q=0$. Suppose that $q \in$ $Q$ for which $\operatorname{Soc}\left(R e_{i}\right) q=0$. Inductively assume that if for $j>i, \operatorname{Soc}\left(R e_{j}\right) p=0$ for $p \in Q$, then $e_{j} p=0$. We have that

$$
\operatorname{Soc}\left(R e_{i}\right)=\bigoplus_{\alpha, \beta} \operatorname{Soc}\left(C_{\beta}^{\alpha}\right)=\bigoplus_{\alpha, \beta}\left[\operatorname{Soc}\left(R e_{i(\alpha)}\right)\right] u_{\beta}^{\alpha} ;
$$

whence [Soc $\left.\left(\operatorname{Re}_{i(\alpha)}\right)\right] u_{\beta}^{\alpha} q=0$ for all $\alpha, \beta$ and thus by the induction hypothesis $e_{i(\alpha)} u_{\beta}^{\alpha} q=u_{\beta}^{\alpha} q=0$. It follows that $E_{m m} q=0$ whenever $u_{\beta}^{\alpha} E_{m m} \neq 0$. Suppose that $e_{i} E_{m m} \neq 0$; then $u_{\beta}^{\alpha} E_{m m} \neq 0$ for some $\alpha, \beta$ since $\Sigma l_{i(\alpha)} v(a)=l_{i}$; as a result we have $E_{m m} q=0$ whenever $e_{i} E_{m m} \neq 0$ and $e_{i} q=0$. Now suppose that $\operatorname{Soc}\left({ }_{R} R\right) q=0$ for $q \in Q$; then $\operatorname{Soc}\left(R e_{i}\right) q=0$ for each $i$, and $e_{i} q=0$ for each $i$. It follows that $q=0$ since $e_{1}+\cdots+e_{n}=1$. We have shown $Z\left(_{R} Q\right)=$ $\operatorname{Ann}_{Q}\left(\operatorname{Soc}\left({ }_{R} R\right)\right)=0$ and ${ }_{R} Q$ is nonsingular. Since ${ }_{Q} Q$ is injective and $Q_{R}$ flat, it follows from Cartan-Eilenberg [4, p. 123, Exercise 10] that ${ }_{R} Q$ is injective. Using the fact that ${ }_{R} Q$ is a nonsingular injective module, we have that $T$ is a subring of $Q$. In fact, $T$ is exactly the subring of $Q$ which leaves $\operatorname{Soc}\left({ }_{R} R\right)$ invariant under right multiplication.

It is clear from the proof that $R$ will be left Artinian if and only if the $v(\alpha)$ 's are integers.

If $R$ is any matrix ring of the form described in Theorem 2.1, then it follows immediately that each $N e_{i}$ is isomorphic to a direct sum of indecomposable left ideals of $R$ and that $R$ is semiprimary. It is then easy to see that $R$ is hereditary. However the theorem gives additional information concerning $Q$ and $R$; namely that $\mathrm{Q}_{R}$ is $R$-flat and that $Q$ contains a copy of the maximal left quotient ring of $R$.

REMARK 2.8. Theorem 2.1 can be restated somewhat. Let $Q$ be the ring of $M \times M$ matrices over $D$. Then $R$ is a semiprimary hereditary basic subring of $Q$ with $Q_{R}$ flat if and only if $R$ is conjugate to a subring of $Q$ having structure (1), (2) and (3). Furthermore, $R$ 's maximal left quotient ring is contained as an overring of $R$ in $Q$ if and only if $\Sigma l_{i(\alpha)} v(a)=l_{i}$ for each $i$. 
The complicated use of flatness in Theorem 2.1 cannot be avoided entirely as is seen by considering the ring $R$ of all matrices of the form $\left[\begin{array}{ll}a & 0 \\ b & c\end{array}\right]$ where $a$ and $c$ are rational numbers while $b$ is a complex number. $R$ is a hereditary semiprimary subring of the ring of 2 by 2 matrices over the complex numbers, $\mathbf{C}_{2}$. But $R$ cannot be put in the form of Theorem 2.1 as a subring of $\mathrm{C}_{2}$ since $R$ is not finite dimensional. This also shows that $\left(\mathrm{C}_{2}\right)_{R}$ is not $R$-flat.

It should be noted that the proof of Theorem 2.1 can be expanded to give an explicit structure for the maximal left quotient ring $T$ of $R$ as an overring of $R$ in $Q$. This also gives the indecomposable nonsingular injective modules over $R$. Since every indecomposable injective module over a semiprimary hereditary ring is a direct summand of a quotient of an indecomposable nonsingular injective module, this gives an explicit structure for indecomposable injectives over $R$. Let us give an outline of that expansion.

Since $T$ is the unique injective hull of ${ }_{R} R$ contained in ${ }_{R} Q, T$ will also be the unique injective hull of $\operatorname{Soc}\left({ }_{R} R\right)$ contained in $Q$; hence we shall first compute $E\left(R e_{j}\right)$ where $R e_{j}$ is simple. By Gordon [16, Theorem 1.2], the $R e_{j}$-homogeneous component of $\operatorname{Soc}\left({ }_{R} R\right)$ is $e_{j} R$; hence $e_{j} R=\bigoplus_{\gamma \in C_{j}} R x_{\gamma}$ where $R x_{\gamma} \simeq R e_{j}$.

Using Theorem 2.1 we can take $R x_{\gamma}$ to be the collection of all matrices of the following form:

$$
\left.B_{\gamma}^{j}=\left[\begin{array}{ccc} 
& 0 \\
\mu_{\gamma} & l_{j} \\
\hdashline 0 & A_{\gamma}^{j} & 0 \\
\hdashline & 0
\end{array}\right]\right\} l_{i<j} l_{i}
$$

where $A_{\gamma}^{j}$ is the collection of all matrices over $D_{j}=e_{j} R e_{j}$; furthermore $x_{\gamma}$ can be taken as the $l_{j} \times l_{j}$ identity matrix. The matrices $\left\{B_{\gamma}^{j}: \gamma \in C_{j}\right\}$ will have nonoverlapping columns for different $\gamma$ 's. Let $D_{\gamma}^{j}$ be the collection of all matrices of the following form:

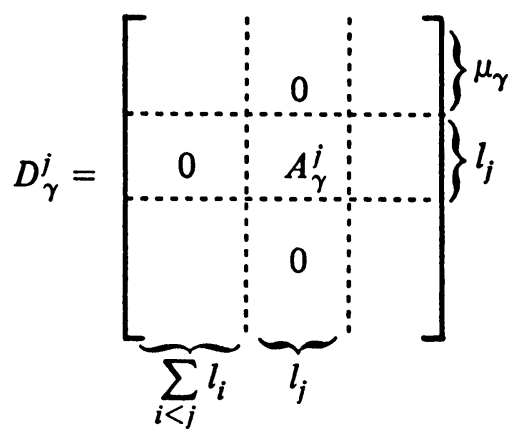


Each element of $D_{\gamma}^{j}$ is almost the transpose of an element of $B_{\gamma}^{j}$.

Let $E^{j}=\Pi_{\gamma \in C_{j}} D_{\gamma}^{j}$. Since the columns of the $B_{\gamma}^{j}$ 's are nonoverlapping, the rows of the $D_{\gamma}^{j}$ 's are nonoverlapping; hence $E^{j}$ can be identified with the obvious subset of $Q$.

Claim. $E^{j}=E\left(R e_{j}\right)$.

Let $y$ be a nonzero element of $E^{j}$, then the $\gamma$ component of $y$ is nonzero for some $\gamma$. In this case $x_{\gamma} y$ will be a nonzero element of $R e_{j}$. Hence $E^{j}$ is essential over $R e_{j}$ and is thus contained in the unique injective hull $E\left(R e_{j}\right)$ contained in $Q$.

Conversely, ${ }_{R} Q e_{j}$ is an injective submodule of ${ }_{R} Q$ which contains $R e_{j}$; therefore, $E\left(R e_{j}\right) \subset Q e_{j}$. Let $y \in E\left(R e_{j}\right)$, then right multiplication by $y$ is an $R$ homomorphism; therefore $x_{\gamma} y \in \operatorname{Soc}\left(E\left(R e_{j}\right)\right)$ for each $\gamma$. As a result, we have that the $A_{\gamma}^{j}$ position of $y$ must be an element of $D_{j}$. Now suppose that $y$ has a nonzero row $\beta$ which is not covered by one of the $A_{\gamma}^{j}$ 's. Since ${ }_{R} Q$ is nonsingular, there is an element $z$ of $\operatorname{Soc}\left({ }_{R} R\right)$ for which $R z$ is simple and for which $z E_{\beta \beta} \neq 0$. Since the $\beta$-row is not covered by an $A_{\gamma}^{j}, R z$ is not isomorphic to $R e_{j}$. As a result we have that $R z y$ is a nonzero simple submodule of $E\left(R e_{j}\right)$ which is not isomorphic to $R e_{j}$. This is a contradiction, and it must be that $y \in E^{j}$ and $E^{j}=E\left(R e_{j}\right)$.

Let $E_{\gamma}^{j}$ be the collection of matrices of the following form:

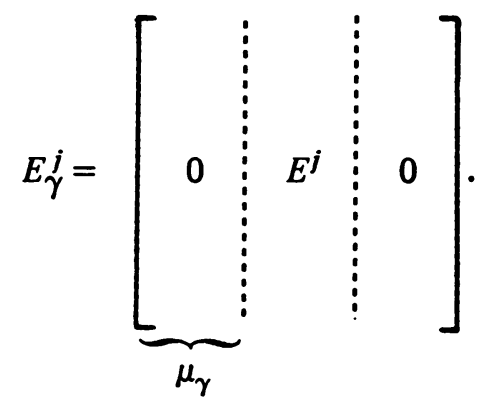

It follows that $E_{\gamma}^{j}=E\left(R x_{\gamma}\right)$. We then have $E\left(e_{j} R\right)=Q \cap\left(\Pi_{\gamma \in C_{j}} E_{\gamma}^{j}\right)$ which is isomorphic (canonically) to the ring of row-finite matrices over the division ring $D_{j}$, as it should be. Doing this for each $j$ for which $R e_{j}$ is simple, and taking the direct sum yields $T$.

EXAMPLE. Let $R$ be the ring of matrices of the form

$$
\left[\begin{array}{lllllll}
a & & & & & \\
0 & a & & & & \\
0 & 0 & a & & & \\
b & 0 & 0 & d & & \\
0 & b & 0 & 0 & d & \\
0 & 0 & c & 0 & 0 & e
\end{array}\right]
$$


where $a \in Q, b, d \in \mathbf{R}$, and $c, e \in \mathbf{C}$. By Theorem 2.1 we have that $R$ is hereditary semiprimary. By the previous discussion $T$ is the ring of matrices of the form

$$
\left[\begin{array}{llllll}
x_{1} & 0 & 0 & x_{2} & 0 & 0 \\
0 & x_{1} & 0 & 0 & x_{2} & 0 \\
0 & 0 & y_{1} & 0 & 0 & y_{2} \\
x_{3} & 0 & 0 & x_{4} & 0 & 0 \\
0 & x_{3} & 0 & 0 & x_{4} & 0 \\
0 & 0 & y_{3} & 0 & 0 & y_{4}
\end{array}\right]
$$

where $x_{i} \in \mathbf{R}, y_{j} \in \mathbf{C}$.

By Theorem 2.1 it would seem that a two-sided hereditary Artinian ring would be very similar to the ring of lower triangular matrices over a division ring and should share many properties with such a ring. The following example shows that one property not shared is the finiteness of indecomposable injective modules.

EXAMPLE. P. M. Cohn [11] has shown that there are division rings $D_{1} \subset$ $D_{2}$ for which $\left[D_{2}: D_{1}\right]_{r}=2$ and $\left[D_{2}: D_{1}\right]_{l}=\infty$. Let

$$
R=\left[\begin{array}{ll}
D_{1} & 0 \\
D_{2} & D_{2}
\end{array}\right] .
$$

The ring $R$ is a left and right Artinian hereditary ring by Theorem 2.1; but

$$
E=E\left(\left[\begin{array}{ll}
0 & 0 \\
0 & D_{2}
\end{array}\right]\right)=\left[\begin{array}{ll}
0 & D_{2} \\
0 & D_{2}
\end{array}\right]
$$

is not finitely generated as a left $R$-module.

In the proof of Theorem 2.1 the structure of $N e_{i}$ was completely determined by the isomorphism type of the semisimple module $N e / N^{2} e_{i}$ (which is given numerically by the $v(\alpha)$ 's). As a result it would be reasonable to conjecture that the diagonal division rings $D_{i}$ together with the isomorphism type of each $N e_{i} / N^{2} e_{i}$ would give a complete set of invariants for basic semiprimary hereditary rings. This is not the case as the following example shows.

EXAMPLE. Let

$$
R=\left\{\left[\begin{array}{ccc}
a+i b & 0 & 0 \\
0 & a+i b & 0 \\
c & d & e
\end{array}\right]\right\} \text { and } S=\left\{\left[\begin{array}{rrr}
a & b & 0 \\
-b & a & 0 \\
c & d & e
\end{array}\right]\right\}
$$

for $a, b \in \mathbf{R} ; c, d, e \in \mathbf{C}$. Each of these rings is a basic hereditary Artinian ring 
having isomorphic diagonal division rings and the same $N e_{i} / N^{2} e_{i}$ structure, but they are not isomorphic (every minimal right ideal of $N(R)$ is a two-sided ideal, while this is not the case for $N(S)$ ).

3. Semihereditary and hereditary rings. In this section we first apply the results of Gordon and Small [18] and Gordon [17] to obtain a structure theorem (Theorem 3.7) for semihereditary rings which are left orders in a semiprimary ring. Our results are motivated by Small's example [29] $R=\left[\begin{array}{ll}8 & 0 \\ Z & Z\end{array}\right]$ of a semihereditary ring which is an order in an Artinian ring. In fact, we show that any semihereditary ring which is a left order in a semiprimary ring is of the same general form.

Then we apply the results of $\S 2$ along with our structure theorem for semihereditary rings which are left orders in a semiprimary ring to obtain a structure theorem for hereditary rings which are left orders in a semiprimary ring. These results differ from those of Small [30], Gordon and Small [18] and Gordon [15] in that the representation is explicit in terms of matrices over left hereditary left Goldie prime rings and their respective classical left quotient rings.

When a semihereditary ring is a two-sided order in a semiprimary ring, our results take on a particularly simplified form. In particular, we obtain Chatters' result [9] that a two-sided hereditary Noetherian ring is a direct sum of an Artinian ring and a semiprime ring. When the maximal quotient ring is two-sided, we recover a result of Goodearl's [13, pp. 38-44].

Before proceeding, we need some terminology. An $R$-module $M$ is said to be torsionfree [25] if for any $x \in M$, the annihilator of $x$ does not contain a regular element of $R$. A ring $R$ with prime radical $N$ is said to satisfy the regularity condition if $a+N$ is regular in $R / N$ implies $a$ is regular in $R$. A ring is said to have enough idempotents [18] if it has a complete set of orthogonal primitive idempotents. Finally, $R$ is said to satisfy the left (right) essentiality condition [17] if $R a(a R)$ is essential in $R$ for every regular element $a$ of $R$.

Gordon [17] gives the following characterization of a left semihereditary ring possessing a semiprimary classical left quotient ring.

THEOREM 3.1. A left semihereditary ring $R$ possesses a semiprimary classical left quotient ring if and only if $R$ has enough idempotents and $R$ satisfies the left essentiality condition.

By Small [32], any left semihereditary ring possessing a semiprimary classical left quotient ring is right semihereditary. Also note that a ring $R$ is a left order in a semiprimary ring $Q$ if and only if $Q$ is the classical left quotient ring of $R$. These facts will be used repeatedly in this section.

Let $R$ be a semihereditary ring possessing a semiprimary classical left quotient ring. Then $R$ is a PWD and has a representation: 


$$
R \simeq\left[\begin{array}{llllll}
P_{1} & & & & & \\
P_{21} & P_{2} & & & & \\
P_{31} & P_{32} & P_{3} & & & \\
\cdot & \cdot & \cdot & \cdot & & \\
\cdot & \cdot & \cdot & \cdot & \\
\cdot & \cdot & \cdot & & \cdot \\
P_{n 1} & P_{n 2} & P_{n 3} & \cdot & \cdot & P_{n}
\end{array}\right]
$$

where each $P_{i}$ is a prime ring and each $P_{i j}$ is a $P_{i}-P_{j}$ bimodule. We will let $e_{k}$ be the matrix unit which has the identity of $P_{k}$ in the $(k, k)$ th block and zeroes elsewhere. Note that $P_{i}=e_{i} R e_{i}$ is semihereditary by [27] and $P_{i j}=e_{i} R e_{j}$.

Since $R$ has a semiprimary classical left quotient ring $Q, R / N$ is a left order in the semisimple ring $Q / N(Q)$.

Lemma 3.2. If $R$ is indecomposable, then $Q$ is indecomposable.

Proof. To show that $Q$ is indecomposable, we need to show that $Q$ has no proper central idempotents. The ring $Q$ is semiprimary and $N(Q)=J(Q)$, so that any orthogonal lifting of a complete set of orthogonal idempotents for $Q / N(Q)$ will be a complete set for $Q$. By Gordon [17, Proposition 1], $R / N(R)$ is a direct sum of uniform left ideals; hence a complete set of orthogonal idempotents for $Q / N(Q)$ may be chosen in $R / N(R)$. The ideal $N(R)$ is a nilpotent ideal of $R$, so that any such set can be lifted to $R$. Therefore any central idempotent of $Q$ belongs to $R$, and $Q$ is indecomposable since $R$ is.

The ring $Q$ is thus an indecomposable hereditary semiprimary ring, and its structure will be given in detail by Theorem 2.1 and the discussion of basic rings in $\S 1$. In general, however, $Q$ will have the following structure:

$$
Q \simeq\left[\begin{array}{llllll}
K_{1} & & & & \\
K_{21} & K_{2} & & & \\
K_{31} & K_{32} & K_{3} & & & \\
\cdot & \cdot & \cdot & \cdot & & \\
\cdot & \cdot & \cdot & \cdot & \\
\cdot & \cdot & \cdot & & \cdot \\
K_{n 1} & K_{n 2} & K_{n 3} & \cdot & K_{n}
\end{array}\right]
$$

Since in the proof of Lemma 3.2 it was shown that a complete set of orthogonal idempotents for $Q$ could be chosen in $R$, we can assume that $K_{i j}=$ 
$e_{i} Q e_{j} \supseteq e_{i} R e_{j}=P_{i j}$. Furthermore, each $P_{i}$ will be a left order in the simple Artinian ring $K_{i}$.

Lemma 3.3. Each $P_{i j}$ is a torsionfree left $P_{i}$-module and a torsionfree right $P_{j}$-module.

Proof. $P_{i j}$ is a right $P_{j}$-submodule of the $K_{j}$-module $K_{i j}$. Since any $K_{j}$ module is torsionfree as a $P_{j}$-module, and since submodules of torsionfree modules are torsionfree, we have that $P_{i j}$ is a torsionfree right $P_{j}$-module. Similarly $P_{i j}$ is a torsionfree left $P_{j}$-module.

LemMA 3.4. Each $P_{i j}$ is a right $K_{j}$-module.

ProOF. Let $e=e_{i}+e_{j}$. The ring $e R e$ is naturally isomorphic to

$$
\left[\begin{array}{cc}
P_{i} & 0 \\
P_{i j} & P_{i}
\end{array}\right]
$$

By [27], $e R e$ is semihereditary. Furthermore $e R e$ is a left order in $e Q e$ [14], so $e R e$ has the left essentiality condition. Hence we may assume that $R=\left[\begin{array}{ll}S & 0 \\ M & T\end{array}\right]$ and show that $M$ is an $S$-divisible right $S$-module. Let $d$ be a regular element of $S$ and suppose that $M d \neq M$. Let $m \in M-M d$ and consider the left ideal

$$
R\left[\begin{array}{ll}
d & 0 \\
m & 0
\end{array}\right]=\left[\begin{array}{cc}
S d & 0 \\
M d+T m & 0
\end{array}\right]
$$

It is not projective, however, as we will show that the natural map $\eta$ of $R$ onto it does not split. Let $K=\operatorname{ker} \eta$; it is easy to see that

$$
K=\left\{\left[\begin{array}{ll}
0 & 0 \\
x & t
\end{array}\right]: x d=-t m\right\} \text {. }
$$

$M_{S}$ is torsionfree by Lemma 3.2. Therefore if

$$
\left[\begin{array}{ll}
0 & 0 \\
x_{1} & t
\end{array}\right] \text { and }\left[\begin{array}{ll}
0 & 0 \\
x_{2} & t
\end{array}\right]
$$

are in $K, x_{1} d=x_{2} d$ or $x_{1}=x_{2}$. Then $K$ is naturally isomorphic to a left ideal of $T$ under the map which sends $\left[\begin{array}{ll}0 & 0 \\ x & t\end{array}\right]$ to $\left[\begin{array}{ll}0 & 0 \\ 0 & t\end{array}\right]$. Call the image of this map $K^{\prime}$.

Since $R$ satisfies the left essentiality condition, $\left[\begin{array}{cc}0 & 0 \\ M d & 0\end{array}\right]$ is essential in $\left[\begin{array}{ll}0 & 0 \\ M & 0\end{array}\right]$ and $M d$ is essential in $M$ as a left $T$-module. Consequently $K^{\prime}=\{t \in T$ : $t m \in M d\}=(m: M d)$ is an essential left ideal of $T$. Note that $1 \notin(m: M d)$ since $m \notin M d$.

The natural map $\eta$ cannot be a splitting map since $K$ is not generated by an 
idempotent; for if $K$ is generated by an idempotent, then $K^{\prime}$ must be generated by an idempotent. But $K^{\prime}$, being essential in $T$, can be generated by an idempotent if and only if the idempotent is the identity of $T$, an impossibility since $1 \notin$ $K^{\prime}$. Hence $M$ is $S$-divisible and $P_{i j}$ is $P_{j}$-divisible.

Now since $P_{i j}$ is a divisible torsionfree $P_{j}$-submodule of the $K_{j}$-module $K_{i j}$, $P_{i j}$ must be $K_{j}$-invariant and hence a right $K_{j}$-module.

The following lemma gives more information concerning the relationship between $R$ and $Q$.

Lemma 3.5. $K_{i j}=K_{i} P_{i j}$.

Proof. Since $K_{i j}=e_{i} Q e_{j}, K_{i}=e_{i} Q e_{i}$, and $P_{i j}=e_{i} R e_{j}$, this is a result of Small as generalized by Gordon [14, Theorem A].

We will also need the following technical lemma.

Lemma 3.6. Let $P$ be a prime left Goldie ring with simple Artinian classical left quotient ring $K$ such that $P \neq K$. Then: (i) $K_{P}$ is not finitely generated; (ii) if $P$ is left Noetherian, then ${ }_{P} K$ is not finitely generated; (iii) if $P$ is left hereditary, then ${ }_{P} K$ is not projective.

Proof. (i) Suppose that $K_{P}$ is finitely generated with generating set $\left\{x_{1}, \ldots, x_{k}\right\}$. A common left denominator $d$ can be chosen so that $x_{i}=d^{-1} a_{i}$ where each $a_{i} \in P$ and $d$ is a regular element of $P$. Then it follows that $d K \in P$, and in particular, $d^{-1}=d d^{-2} \in P$. This is a contradiction since $P \neq K$.

(ii) Let $d$ be a regular element in $P$ for which $d^{-1} \notin P$. If ${ }_{P} K$ is finitely generated, then ${ }_{P} K$ will be Noetherian since $P$ is left Noetherian. Hence there is a positive integer $m$ for which $P d^{-m}=P d^{-(m+1)}$. Then there exists an $x \in P$ for which $x d^{-m}=d^{-(m+1)}$; we then have $d^{-1}=x$ an element of $P$, which is a contradiction.

(iii) Since ${ }_{P} P$ has finite Goldie dimension, it follows from Albrecht [1] that $P$ is left Noetherian. If ${ }_{P} K$ is projective, then ${ }_{P} K$ will be finitely generated by Albrecht [1] since it also must have finite Goldie dimension. This contradicts (ii).

If $\left\{e_{1}, e_{2}, \ldots, e_{n}\right\}$ is an ordered set of orthogonal idempotents for a ring $R$, for notational purposes we will let $f_{i}=e_{i}+\cdots+e_{n}$.

We are now in a position to state the main theorem on semihereditary rings. If $R$ has a semiprimary classical quotient ring, then $R$ will be a direct sum of indecomposable rings. Hence it is sufficient in the following theorem to assume that $R$ is indecomposable.

THEOREM 3.7. Let $R$ be an indecomposable semihereditary ring with semiprimary classical left quotient ring $Q$; let $R$ and $Q$ have general form as in (1) and (2). Then each $P_{i}$ is a semihereditary left Goldie prime ring, each $P_{i j}$ is a 
right $K_{j}$-module, and for each $i, f_{i} R e_{i-1}$ is a flat left $f_{i} R f_{i}$-module.

Conversely, any such ring is semihereditary.

Proof. By Gordon and Small [18] each $P_{i}$ is a semihereditary prime left Goldie ring, while Lemma 3.4 shows that $P_{i j}$ is a right $K_{j}$-module. It remains to be shown that $f_{i} R e_{i-1}$ is a flat left $f_{i} R f_{i}$-module for $i=2, \ldots, n$.

The ring $f_{i-1} R f_{i-1}$ is again semihereditary by [27], so it is sufficient to assume that $R$ is of the form $R=\left[\begin{array}{ll}S & 0 \\ M & T\end{array}\right]$, and then show that ${ }_{T} M$ is flat. But ${ }_{R} M={ }_{T} M$; therefore since ${ }_{R} M$ is flat, ${ }_{T} M$ is also flat.

To prove the converse we need only show that $R$ is semihereditary. To do this we induct on $n$, the number of orthogonal central idempotents of $Q / N(Q)$. If $n=1$, the result is trivial. Now assume the result holds for $n=r-1$ and let

$$
W=\left[\begin{array}{llll}
P_{1} & & \\
P_{21} & P_{2} & 0 \\
\cdot & \cdot & \cdot & \\
\cdot & \cdot & \cdot & \\
\cdot & \cdot & & \cdot \\
P_{r 1} & P_{r 2} & \cdot & \cdot
\end{array}\right]
$$

satisfy the conditions of the theorem.

Let

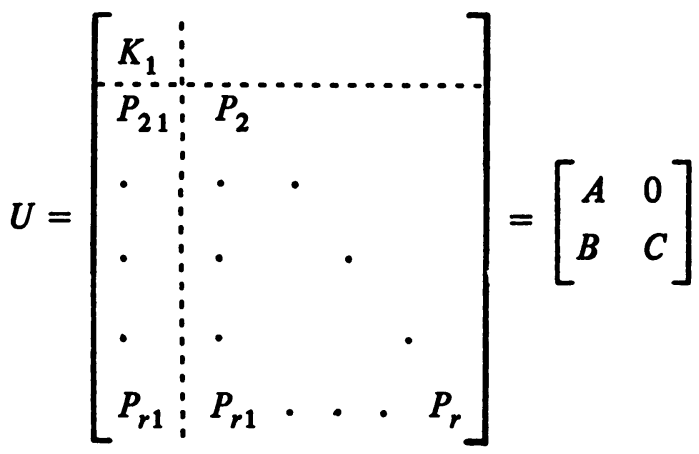

be partitioned as indicated. By the assumptions of the theorem, $c^{B}$ is flat; hence by Fields $[12$, p. 348] and the induction hypothesis we have that $\operatorname{WGD}(U) \leqslant 1$. Since $Q$ is semiprimary, it follows from Jondrup [23, Corollary 3.2] that finitely generated flat $U$-modules are projective; whence $U$ is semihereditary.

Now let 


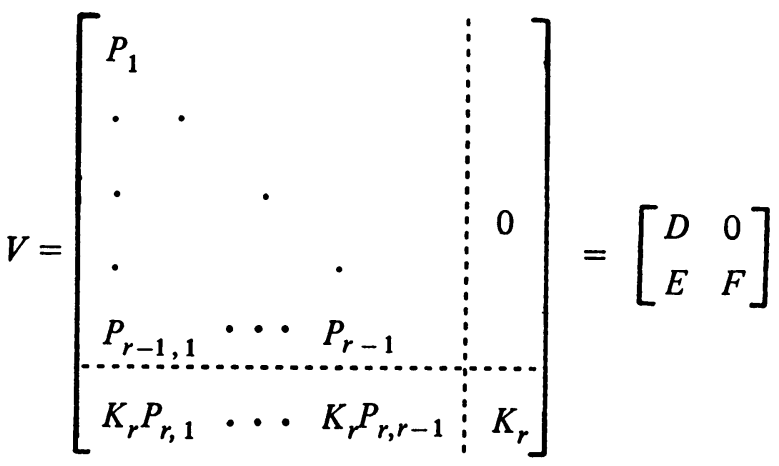

be partitioned as indicated. Let

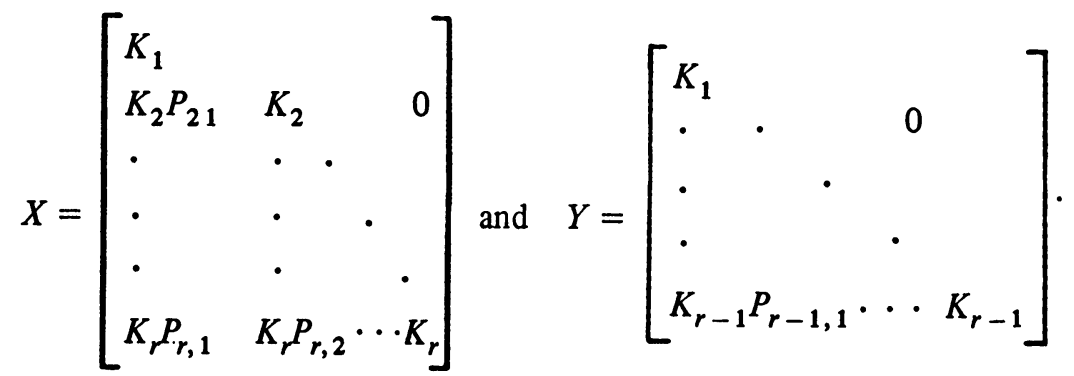

Now $Y$ is the classical left quotient ring of $D$ so $Y_{D}$ is flat. $E$ is a right ideal of $X$, and $X$ is hereditary; hence $E_{X}$ is flat. However $E_{X}=E_{Y}$, so $E_{Y}$ is flat. Then by $\left[4\right.$, Problem 10, p. 123], $E_{D}$ is flat. Again by [12, p. 348], the induction hypothesis, and [23, Corollary 3.2], it follows that $V$ is semihereditary.

Since $W$ has a semiprimary classical left quotient ring $X$, to show $W$ is semihereditary it suffices to show $W$ is right semihereditary [32]. To do this, it is sufficient to show that any finitely generated right ideal $I_{W}$ contained in $e_{k} W$ is projective for $1 \leqslant k \leqslant r$. If $1 \leqslant k<r, e_{k} W=e_{k} V$. Since $V$ is semihereditary, $I_{V}$ is projective so $I_{W}$ is projective. Now let $I_{W}$ be a submodule of $e_{r} W$ and let $I=\sum_{k=1}^{m} x_{k} W$ where $e_{r} x_{k} e_{1}=0$ for $k=1, \ldots, t$ and $e_{r} x_{k} e_{1}=x_{k}$ for $k=$ $t+1, \ldots, m$. If $j \leqslant t$, then $x_{j} W=x_{j} U$. Let $J=\Sigma_{k=1}^{t} x_{k} W=\Sigma_{k=1}^{t} x_{k} U$. $U$ is semihereditary, so $J_{U}$ is projective. Let $P=\Sigma_{k>j} e_{k} W$ and let $T_{U}$ be the trace of $P$ in $J$. If $T_{U} \neq J$, then $\operatorname{Hom}_{U}(P, J / T)=0$ since $P_{U}$ is projective. Therefore $J / T \simeq \operatorname{Hom}_{U}(U, J / T) \simeq \operatorname{Hom}_{U}(P, J / T) \oplus \operatorname{Hom}_{U}\left(K_{1}, J / T\right)=\operatorname{Hom}_{U}\left(K_{1}, J / T\right)$.

Hence $J / T$ is a semisimple module of homogeneous type $K_{1}$, and consequently is $U$-projective. Therefore $J \simeq T \oplus J / T$ and $J$ has a direct summand isomorphic to $K_{1}$. If $P_{1} \neq K_{1}$, then $\left(K_{1}\right)_{P_{1}}$ is not finitely generated by Lemma 3.6 (if $P_{1}=$ $K_{1}$, then $W=U$ is already semihereditary); hence we have a contradiction to the fact that $J_{W}$ is finitely generated. Hence $T=J$ and $J$ being $U$-projective is a direct summand of a direct sum of copies of $P ; J$ is thus $W$-projective. 
Now $I e_{1}$ has the same right $W$-structure as it has right $P_{1}$ structure. $J e_{1}$ is a right $U$-submodule of $J$; hence it is a module over $K_{1}$. Since $K_{1}$ is semisimple, $J e_{1}$ is a direct summand of the $K_{1}$-module $P_{r 1}$. Since $P_{1}$ is a subring of $K_{1}, J e_{1}$ is a $P_{1}$ direct summand of any submodule of $P_{r 1}$ containing $J e_{1}$. In particular, $J e_{1}$ is a direct summand of $I e_{1}$; so $I e_{1}=J e_{1} \oplus L$ for some submodule $L$ of $I e_{1}$. But $I=J+L$ and $J \cap L=J e_{1} \cap L=0$, so $I=J \oplus L$. $V$ is semihereditary so $L$ is $V$-projective. Therefore $L$ is $P_{1}$-projective and hence $W$-projective. Finally we have $I_{W}$ is projective and $W$ is semihereditary. Hence it follows that $R$ is semihereditary.

REMARK. Note that many of our arguments used only the fact that $R$ was a (left) p.p. ring with semiprimary classical left quotient ring $Q$. In fact, assuming that $R$ is (left) p.p. and that $R$ and $Q$ are as in (1) and (2), then each $P_{i}$ is a left p.p. Goldie prime ring, each $P_{i j}$ is a right $K_{j}$-module and for each $i, f_{i} R e_{i-1}$ has projective left principal $f_{i} R f_{i}$-submodules. In particular this may shed some light on Gordon's question[14] about left p.p. rings possessing a semiprimary classical left quotient ring.

If $R$ is a semihereditary PWD, then the $P_{i j}$ 's are flat left $P_{i}$-modules. This can be seen by considering the subring $e R e$ where $e=e_{i}+e_{j}$ and recalling that $e R e$ is semihereditary. Unfortunately the condition in Theorem $3.7, f_{i} R e_{i-1}$ is a flat left $f_{i} R f_{i}$-module, cannot be replaced by the simpler condition, $P_{i j}$ is a flat left $P_{i}$-module, as seen by the following example. Let $\mathbf{C}_{(x)}[x]$ be the localization of the polynomial ring $\mathbf{C}[x]$ over the complex numbers at the prime ideal $(x)$. Then $\mathbf{C}_{(x)}[x]$ will be a local principal ideal ring with completion the ring of formal power series over C. Then by Kaplansky [24, p. 46] there exists an indecomposable torsionfree module $M$ of rank two over $\mathbf{C}_{(x)}[x]$. Now let

$$
R=\left[\begin{array}{llc}
\mathrm{R} & 0 & 0 \\
\mathrm{C} & \mathrm{R} & 0 \\
M & M & \mathrm{C}_{(x)}[x]
\end{array}\right]
$$

where $\mathbf{R}$ denotes the field of real numbers. The ring $R$ has a semiprimary classical left quotient ring and the respective $P_{i j}$ 's are flat $P_{i}$-modules, but the ring is not semihereditary. To see this, note that $\left[{ }_{M}^{C}\right]$ needs two generators over the ring

$$
S=\left[\begin{array}{cc}
\mathrm{R} & 0 \\
M & \mathrm{C}_{(x)[x]}
\end{array}\right]
$$

and hence $\left[{ }_{M}^{\mathrm{C}}\right.$ ] cannot be $S$-flat (equivalently projective) since it is indecomposable. Consequently $R$ is not semihereditary.

Notice that the ring $S$ in the previous paragraph is a semihereditary ring by Theorem 3.7; however, $M$ is not a direct sum of uniform $\mathbf{C}_{(x)}[x]$ ideals. If $R$ 
is a left hereditary ring possessing a semiprimary classical left quotient ring, we will show that this cannot occur.

Let $R$ be a semihereditary ring with semiprimary classical left quotient ring $Q$ as in (1) and (2). Now let $e_{i}=e_{i 1}+\cdots+e_{i \mu(i)}$ where $\mu(i)$ is a positive integer for $i=1, \ldots, n$ and each $e_{i j}$ is a primitive idempotent of $Q$. The following lemma shows that we can reduce the study of projective modules in our situation to indecomposable projective modules.

LEMMA 3.8. Let $R$ and $Q$ be as in the previous paragraph. If $I$ is a finitely generated indecomposable left ideal of $R$, then $Q I$ is an indecomposable projective $Q$-module. Furthermore, every projective $R$-module is a direct sum of indecomposable finitely generated projective modules.

Proof. To prove the first statement, let $I$ be a finitely generated indecomposable left ideal of $R$ and let $i_{0}$ be the largest $i$ for which there is a nonzero projection of $I$ into $R e_{i j}$. Since $I$ is indecomposable, there is an $l$ such that $I$ projects monomorphically into $R e_{i_{0} l}$. Identify $I$ with this image. Then $Q I \subset$ $Q R e_{i_{0} l}=Q e_{i_{0} l}$. Since $e_{i_{0}} I \neq 0$ (otherwise a larger $i$ could have been chosen), $Q I \not N(Q) e_{i_{0} l}$ so $Q I=Q e_{i_{0} l}$ is indecomposable.

To prove the second statement, by Albrecht [1] each projective left $R$-module is isomorphic to a direct sum of finitely generated left ideals of $R$; therefore it is sufficient to show that each finitely generated left ideal is a direct sum of indecomposable left ideals. Let $I$ be a finitely generated left ideal of $R$ and let the number of indecomposable direct summands of $Q I$ be $k$. The integer $k$ is uniquely determined since $Q$ is semiprimary. Suppose $I=I_{1} \oplus \cdots \oplus I_{t}$ where each $I_{j} \neq 0$. Then by [31, Corollary 1.6], $Q I=Q I_{1} \oplus Q I_{2} \oplus \cdots \oplus Q I_{t}$. Thus $t \leqslant k$ and $I$ can be written as a finite direct sum of indecomposable finitely generated left ideals.

The next proposition is a key result in that it characterizes indecomposable projective modules over left hereditary PWD's possessing a semiprimary classical left quotient ring.

Proposition 3.9. Let $R$ be a left hereditary ring possessing a semiprimary classical left quotient ring $Q$ with structure as in (1) and (2). If $F$ is an indecomposable projective left $R$-module, then

$$
F \simeq{ }^{t}\left[U_{\alpha}: N(R) e_{\alpha 1}\right]
$$

where $U_{\alpha}$ is a finitely generated uniform projective submodule of $P_{\alpha} K_{\alpha}$ for some $\alpha$.

Proof. By Lemma 3.8 and a simple induction on the triangular structure of $R$, we may assume without loss of generality that $F$ is a finitely generated submodule of $Q e_{11}$ for which $e_{1} F \neq 0$; i.e., $F \not \subset N(Q) e_{11}$. 
Since $e_{1} F \neq 0$ and $e_{1} F$ is a submodule of the uniform $P_{1}$-module $e_{1} Q e_{11}$, we may take $U$ to be $e_{1} F$. Hence $R\left[\begin{array}{l}U \\ 0\end{array}\right] \subset F$; that is,

$$
{ }^{t}\left[U: P_{21} U: \cdots: P_{21} U\right] \subset F .
$$

Now by Lemma 3.4, $P_{i 1}$ is a right $K_{1}$-module; therefore $P_{i 1}=P_{i 1} K_{1} U=$ $P_{i 1} K_{1} e_{11}=P_{i 1} e_{1}$. Thus $N(R) e_{11} \subset F$. Letting $f_{2}$ be as in Theorem 3.7, we have $N(R) e_{11} \subset f_{2} F$. We claim that $N(R) e_{11}=f_{2} F$.

Le:t $x \in f_{2} F$; we want to show that $x \in N(R) e_{11}$. Since $P_{1}$ is a prime ring, $e_{11} F \cap P_{1} \neq 0$ for otherwise $e_{1} F \cap P_{1}$ is a nilpotent left ideal of $P_{1}$. So let $y$ be a nonzero element of $e_{11} F \cap P_{1}$ and then let $z=x+y$; note that $x=f_{2} z$. $R z$ is a cyclic submodule of the projective $R$-module $F$; hence $R z$ is projective. As before $N(R) e_{11} \subset R z$ and $y \neq 0$; therefore $R z$ must be indecomposable. Thus $R z$ is isomorphic to a principal left ideal $I$ of $R e_{1}$. Let the image of $z$ under this isomorphism be $w$. Since $e_{11} z \neq 0, e_{11} w \neq 0$ so $w=\left[\begin{array}{ll}a & 0 \\ b & 0\end{array}\right]$ where $R$ is partitioned as

$$
R=\left[\begin{array}{cc}
e_{1} R e_{1} & 0 \\
f_{2} R e_{1} & f_{2} R f_{2}
\end{array}\right]
$$

Since $e_{11} a \neq 0$, there exists an $i$ such that $e_{11} a e_{1 i} \neq 0$. By Gordon [17, Theorem 2] there exists $0 \neq u \in e_{1 i} R e_{11}$. Then $a u=w u \neq 0$ since $R$ is a PWD; so $R w u$ and $P_{1} a u$ are nonzero homomorphic images of $R w$ and $P_{1} a$ respectively. Since $R w u$ is projective and both $R w$ and $P_{1} a$ are indecomposable, it follows that $R w u \simeq R w$ and $P_{1} a u \simeq P_{1} a$. Thus we may assume that $e_{11} a e_{11}=a$.

Now $x \in f_{2} Q e_{1} \backslash f_{2} R e_{1}$, so $x=d^{-1} c$ for $d$ regular in $f_{2} R f_{2}, c \in f_{2} R e_{1}$. Since $x \notin R, c \notin d f_{2} R e_{1}$. Now $z=\left[\begin{array}{ll}y & 0 \\ x & 0\end{array}\right]$ and $N(R) e_{11} \subset R y$. In particular, there is a $p \in f_{2} R e_{1}$ for which $p y=c$. Note that $p \notin d_{2} f_{2} R e_{1}$ since $c \notin$ $d f_{2} R e_{1}$. Now

$$
\left[\begin{array}{rr}
0 & 0 \\
p & -d
\end{array}\right]\left[\begin{array}{cc}
y & 0 \\
d^{-1} c & 0
\end{array}\right]=\left[\begin{array}{cc}
0 & 0 \\
p y-d d^{-1} c & 0
\end{array}\right]=\left[\begin{array}{ll}
0 & 0 \\
0 & 0
\end{array}\right]
$$

and

$$
\left[\begin{array}{rr}
0 & 0 \\
p & -d
\end{array}\right]\left[\begin{array}{ll}
a & 0 \\
b & 0
\end{array}\right]=\left[\begin{array}{cc}
0 & 0 \\
p a-d b & 0
\end{array}\right] \text {. }
$$

Since $d f_{2} R e_{1}$ is a right $K_{1}$-module, $d f_{2} R e_{11}$ is a right $e_{11} K_{1} e_{11}=D_{1}$-module. Thus if $p a \in d f_{2} R e_{11}$, we must have $p e_{11} a e_{11}=p a \in d f_{2} R e_{11}$. Since the elements of $D_{1}=e_{11} K_{1} e_{11}$ are invertible, $p \in d f_{2} R e_{11}$, a contradiction. Thus $x \in f_{2} R e_{1}$ as desired.

Let $P$ be a prime left hereditary left Goldie ring with classical left quotient ring $K$ and $e$ a primitive idempotent of $R$. A finitely generated submodule of 
$K e$ containing $P e$ will be called a uniform fractional left P-ideal.

We now state our main structure theorem for left hereditary PWD's which are left orders in a semiprimary classical left quotient ring. The $P_{i}$ 's, $P_{i j}$ 's, $K_{i}$ 's, $K_{i j}$ 's and $e_{i j}$ 's are as defined in this section.

THEOREM 3.10. Let $R$ be an indecomposable, left hereditary ring possessing a semiprimary classical left quotient ring $Q$ with structure as in (1) and (2). Then

(a) each $P_{i}$ is a left hereditary, left Noetherian prime ring;

(b) each $P_{i j}$ is a right $K_{j}$-module;

(c) there is a sequence of integers $i<i(1)<\cdots<i(k) \leqslant n$ and a sequence of ordinal numbers $v(1), \ldots, v(k)$ such that

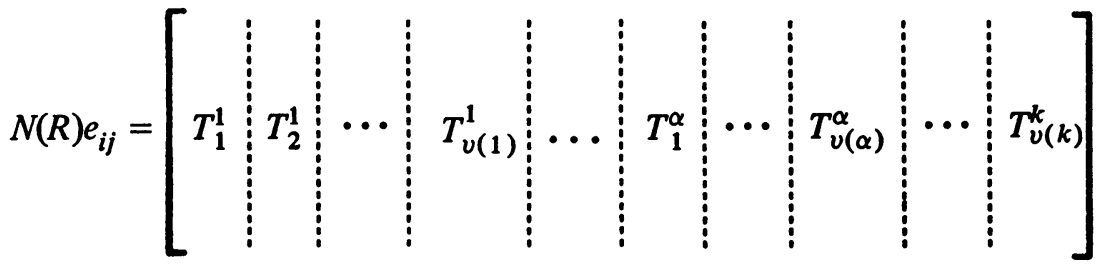

where

$$
T_{\alpha}^{\beta}={ }^{t}\left[0: U_{i(\alpha)} \vdots N(R) e_{i(\alpha)}\right]
$$

and $U_{i(\alpha)}$ is a uniform projective fractional left $P_{i(\alpha)}$-ideal.

Conversely, any such matrix ring is a left hereditary ring possessing a semiprimary classical left quotient ring.

Proof. As in the proof of Theorem 3.7, $P_{i}=e_{i} R e_{i}$ is a left hereditary prime Goldie ring, so $P_{i}$ is left Noetherian by Levy [25]. By Lemma $3.4, P_{i j}$ is a right $K_{j}$-module.

Now consider $N(R) e_{i j} . R$ is left hereditary so $N(R) e_{i j}$ is projective; hence $N(R) e_{i j}=\bigoplus_{\delta \in \Delta} I_{\delta}$ where each $I_{\delta}$ is a finitely generated indecomposable projective $R$-module. Then $N(Q) e_{i j}=Q N(R) e_{i j}=\bigoplus_{\delta \in \Delta} Q I_{\delta}$, where each $Q I_{\delta}$ will be indecomposable and $Q$-projective by Lemma 3.8. Thus $Q I_{\delta}$ is isomorphic to $Q e^{\delta}$ for some primitive idempotent $e^{\delta}$ of $Q$. Since $e^{\delta} Q I_{\delta} \neq 0, e^{\delta} I_{\delta} \neq 0$ so $e^{\delta} I_{\delta}$ is isomorphic to a uniform submodule of $K_{\delta} e^{\delta}$. Letting $u^{\delta} \neq 0 \in e^{\delta} I_{\delta}$, it follows from the proof of Proposition 3.9 that $Q u^{\delta} \simeq Q e^{\delta}=Q I_{\delta}$. As in Theorem 2.1, a "new basis" can be chosen so that each $u^{\delta}$ is a small identity matrix. Furthermore, there are integers $i<i(1)<\cdots<i(k) \leqslant n$ and ordinal number $v(1), \ldots, v(k)$ such that the $u^{\delta}$ 's can be indexed as $u_{\beta}^{\alpha}$ 's as in Theorem 2.1. In this case

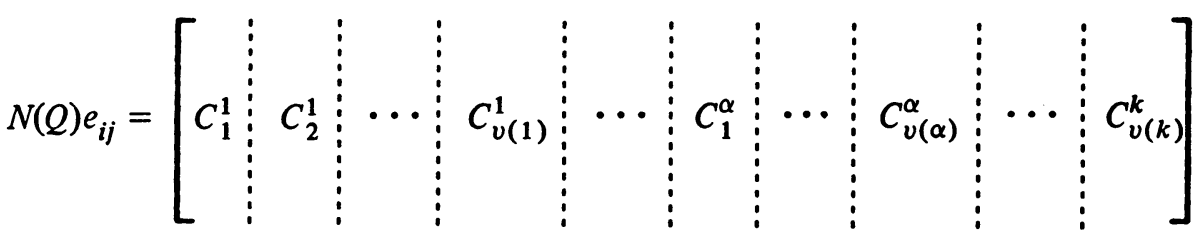


where each $C_{\beta}^{\alpha}$ is the collection of matrices of the form

$$
{ }^{t}\left[0 \vdots Q e_{i(\alpha) 1}\right] \text {. }
$$

This induces a representation for $R$ so that

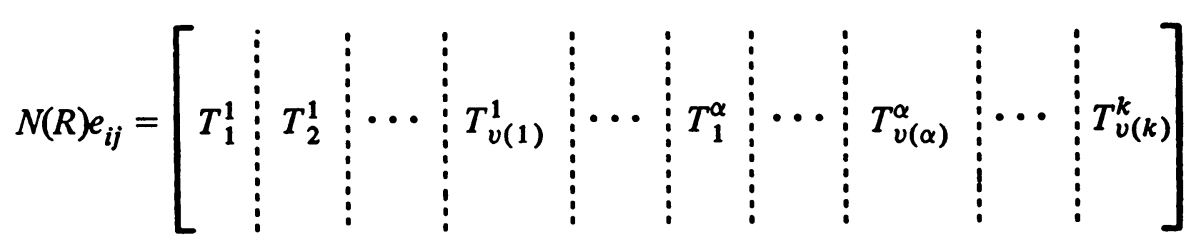

where each $T_{\beta}^{\alpha}$ is a finitely generated indecomposable projective submodule of $C_{\beta}^{\alpha}$ containing $R e_{i(\alpha)}$. Then by Proposition 3.9,

$$
T_{\alpha}^{\beta}={ }^{t}\left[0 \vdots U_{i(\alpha)}: N(R) e_{i(\alpha) 1}\right]
$$

where $U_{i(\alpha)}$ is a uniform projective fractional left $P_{i(\alpha)}$-ideal.

To prove the converse, suppose $R$ and $Q$ have the indicated structure. As in the proof of the converse of Theorem 3.7, we induct on the number of central idempotents of $R / N$. If $n=1$, the result is trivial. Now assume that the result holds for $k<n$. Let $I$ be a left ideal of $R$ and show that $I$ is projective. We may assume that $I \subset R e_{i j}$. If $I \subset N(R) e_{i j}$, then $I$ is a projective left $f_{i+1} R f_{i+1}$-module by the induction hypothesis when $f_{i+1}=e_{i+1}+\cdots+e_{n}$; hence $I$ is $R$-projective. If $I \not \subset N(R) e_{i j}$, then $e_{i} I$ is a uniform left $P_{i}$-ideal. Hence by the proof of Proposition 3.9, $N(R) e_{i j} \subset I$ and $I / N(R) e_{i j}$ is finitely generated since $P_{i}$ is left Noetherian. But $N(R) e_{i j}$ is small in $I$ so $I$ is finitely generated. Therefore, $I$ is projective, since by Theorem 3.7, $R$ is semihereditary. Thus $R$ is left hereditary.

The following example illustrates the power of Theorem 3.10.

ExAMPLE. Let $F$ be a field, $F[x]$ the ring of polynomials over $F$ and $F(x)$ the quotient field of $F[x]$. Then the ring

$$
R=\left[\begin{array}{ccc}
F & 0 & 0 \\
F & F & 0 \\
F[x] \frac{1}{x} & F[x] & F[x]
\end{array}\right]
$$

has a semiprimary classical left quotient ring

$$
\left[\begin{array}{ccc}
F & 0 & 0 \\
F & F & 0 \\
F(x) & F(x) & F(x)
\end{array}\right]
$$


However, by Theorem $3.11, R$ is not left hereditary since $N(R) e_{1}$ is not the set of matrices over $R e_{2}$. Note that the ring

$$
\left[\begin{array}{ccc}
F & 0 & 0 \\
F & F & 0 \\
F[x] \frac{1}{x} & F[x] \frac{1}{x} & F[x]
\end{array}\right]
$$

is left hereditary.

We now shift our direction towards two-sided classical quotient rings. In case a semihereditary ring possesses a semiprimary classical two-sided quotient ring, then every flat left $Q$-module is also $R$-flat. Thus Theorem 3.7 reduces to

THEOREM 3.11. Let $R$ be an indecomposable semihereditary ring with semiprimary classical two-sided quotient ring $Q$. Then each $P_{i}$ is a semihereditary two-sided Goldie prime ring with two-sided classical quotient ring $K_{i}$ and $P_{i j}=K_{i j}$. Conversely, any such ring is semihereditary.

REMARK. Theorem 3.11 gives a surprisingly complete characterization of a class of semihereditary rings including the class of two-sided finite dimensional rings; it basically says that such a ring is a hereditary semiprimary ring except that prime rings are sprinkled along the diagonal.

Now let $R$ be a two-sided hereditary ring which is a two-sided order in a semiprimary ring. Then by Lemma 3.6, $K_{i}$ cannot be a projective left or right $P_{i}$-module unless $P_{i}=K_{i}$. Hence it follows that $K_{i j}=0$ whenever $P_{i} \neq K_{i}$ or $P_{j}$ $\neq K_{j}$. Thus we can order the $P_{i}$ 's such that $R \simeq\left[\begin{array}{ll}A & 0 \\ 0 & S\end{array}\right]$ where $A$ is a semiprimary ring and $S$ is a direct sum of prime rings. Putting all of this together, we have

THEOREM 3.12. A (two-sided) hereditary ring which is a two-sided order in a semiprimary ring is a direct sum of a semiprimary ring and a semiprime ring.

As a corollary we have a new proof of Chatters' result [9] which does not use the restricted minimum condition.

Corollary 3.13. A hereditary Noetherian ring is a direct sum of an Artinian ring and a direct sum of prime rings.

Finally we consider the situation when a semihereditary ring with semiprimary classical left quotient ring has a two-sided maximal quotient ring. Part (i) of the following theorem has already been proved by Goodearl [13]; however, our proof is somewhat shorter than his. 
THEOREM 3.14. Let $R$ be an indecomposable semihereditary ring with semiprimary classical left quotient ring $Q$. If $R$ has a two-sided maximal quotient ring $T$, then

(i) $Q$ is Morita equivalent to a full block lower triangular matrix ring over a division ring (and hence is a hereditary serial ring); and

(ii) each $P_{i}$ is a two-sided order in $K_{i}$ and $K_{i j}=P_{i j}$.

Proof. First we show (i). As in $\S 2, T$ will be a direct sum of full linear rings over division rings. Since $T$ is the two-sided maximal quotient ring of $R, T$ will be left and right self-injective. Since a full linear ring is two-sided self-injective if and only if it is semisimple (Osofsky [26]) and $Q$ is semiprimary, we have that $Q$ is right and left Artinian by Sandomierski [28]. Since by Cateforis [6], a semisimple maximal left quotient ring is two-sided if and only if injective hulls of finitely generated projective modules are flat, it will be sufficient to assume that $Q$ is basic. In that case the structure of $Q$ is given by Theorem 2.1. In terms of the notation of Theorem 2.1 , by the left-hand version of Theorem 2.1, $\cdots l_{1} \geqslant l_{2} \geqslant \cdots \geqslant l_{n}$ and by the right-hand version of Theorem 2.1, $l_{n} \geqslant l_{n-1} \geqslant \cdots \geqslant l_{1}$. Thus $l_{1}=l_{2}=\cdots=l_{n}$. By Proposition 2.7, the ranks for a family of generators for $N e_{i}$ must be $l_{i}$; hence it must be that $Q e_{n}$ is the only projective simple left module and each $Q e_{i}$ has simple socle. Thus it follows that $Q$ is a lower block matrix ring over some division ring.

To prove (ii) note that since the maximal quotient ring $T$ of $R$ is two-sided, $T$ must be the two-sided maximal quotient ring of $Q$ also. Hence $T$ is semisimple and $Q$ is a hereditary serial ring by the first part of the proof. It follows that $R_{R}$ must have finite Goldie dimension and $R$ is a two-sided order in $Q$. Then the desired structure follows from Theorem 3.11.

ACKNowledgement. The authors would like to express their gratitude to the referee for a number of helpful suggestions.

\section{REFERENCES}

1. F. Albrecht, On projective modules over semi-hereditary rings, Proc. Amer. Math. Soc. 12 (1961), 638-639. MR 23 \#A3766.

2. M. Auslander, On the dimension of modules and algebras. III. Global dimension, Nagoya Math. J. 9 (1955), 67-77. MR 17, 579.

3. H. Bass, Finitistic dimension and a homological generalization of semi-primary rings, Trans. Amer. Math. Soc. 95 (1960), 466-488. MR 28 \#1212.

4. H. Cartan and S. Eilenberg, Homological algebra, Princeton Univ. Press, Princeton, N. J., 1956. MR 17, 1040.

5. V. C. Cateforis, Flat regular quotient rings, Trans. Amer. Math. Soc. 138 (1969), 241-249. MR 39 \#259.

6. - Two-sided semisimple maximal quotient rings, Trans. Amer. Math. Soc. 149 (1970), 339-349. MR 41 \#5424.

7. S. U. Chase, Direct products of modules, Trans. Amer. Math. Soc. 97 (1960), 457473. MR 22 \#11017. 
8. S. U. Chase, $A$ generalization of the ring of triangular matrices, Nagoya Math. J. 18 (1961), 13-25. MR 23 \#A919.

9. A. W. Chatters, A decomposition theorem for Noetherian hereditary rings, Bull. London Math. Soc. 4 (1972), 125-126. MR 47 \#8607.

10. P. M. Cohn, The embedding of firs in skew fields, Proc. London Math. Soc. (3) 23 (1971), 193-213. MR 45 \#6866.

11. Q Quadratic extensions of skew fields, Proc. London Math. Soc. (3) 11 (1961), 531-556. MR 25 \#101.

12. K. L. Fields, On the global dimension of residue rings, Pacific J. Math. 32 (1970), 345-349. MR 42 \#6049.

13. K. R. Goodearl, Singular torsion and the splitting properties, Mem. Amer. Math. Soc. No. 124 (1972).

14. R. Gordon, Classical quotient rings of PWD's, Proc. Amer. Math. Soc. 36 (1972), 39-46. MR 46 \#9086.

15. - Rings defined by R-sets and a characterization of a class of semiperfect rings, Trans. Amer. Math. Soc. 155 (1971), 1-17. MR 42 \#7706.

16. , Rings in which minimal left ideals are projective, Pacific J. Math. 31 (1969), 679-692. MR 42 \#314.

17. - Semi-prime right Goldie rings which are direct sums of uniform right ideals, Bull. London Math. Soc. 3 (1971), 277-282. MB 45 \#3473.

18. R. Gordon and L. W. Small, Piecewise domains, J. Algebra 23 (1972), 553-564. MR 46 \#9087.

19. M. Harada, Hereditary semi-primary rings and trianguiar matrix rings, Nagoya Math. J. 27 (1966), 463-484. MR 34 \#4300.

20. N. Jacobson, Lectures in abstract algebra. II. Linear algebra, Van Nostrand, Princeton, N. J., 1953. MR 14, 837.

21. R. E. Johnson, The extended centralizer of a ring over a module, Proc. Amer. Math. Soc. 2 (1951), 891-895. MR 13, 618.

22. - Quotient rings of rings with zero singular ideal, Pacific J. Math. 11 (1961), 1385-1392. MR 26 \#1331.

23. S. Jфndrup, On finitely generated flat modules. II, Math. Scand. 27 (1970), 105112. MR 43 \#3298.

24. I. Kaplansky, Infinite abelian groups, Univ. of Michigan Press, Ann Arbor, Mich., 1954. MR 16, 444.

25. L. Levy, Torsion-free and divisible modules over non-integral-domains, Canad. J. Math. 15 (1963), 132-151. MR 26 \#155.

26. B. Osofsky, Cyclic injective modules of full linear rings, Proc. Amer. Math. Soc. 17 (1966), 247-253. MR 32 \#7604.

27. F. L. Sandomierski, $A$ note on the global dimension of subrings, Proc. Amer. Math. Soc. 23 (1969), 478-480. MR 39 \#6930.

28. , Semisimple maximal quotient rings, Trans. Amer. Math. Soc. 128 (1967), 112-120. MR $35 \# 5473$.

29. L. W. Small, An example in Noetherian rings, Proc. Nat. Acad. Sci. U.S.A. 54 (1965), 1035-1036. MR 32 \#561. \#178.

30. - Hereditary rings, Proc. Nat. Acad. Sci. U.S.A. 55 (1966), 25-27. MR 32

31. - Orders in Artinian rings, J. Algebra 4 (1966), 13-41. MR 34 \#199.

32. - Semihereditary rings, Bull. Amer. Math. Soc. 73 (1967), 656-658.

MR 35 \#2926.

\section{DEPARTMENT OF MATHEMATICS, UNIVERSITY OF NORTHERN COLORADO, GREELEY, COLORADO 80639}

DEPARTMENT OF MATHEMATICS, WAKE FOREST UNIVERSITY, WINSTONSALEM, NORTH CAROLINA 27109 